\title{
Time-Frequency ARMA Models and Parameter Estimators for Underspread Nonstationary Random Processes
}

\author{
Michael Jachan, Gerald Matz, Senior Member, IEEE, and Franz Hlawatsch, Senior Member, IEEE
}

\begin{abstract}
Parsimonious parametric models for nonstationary random processes are useful in many applications. Here, we consider a nonstationary extension of the classical autoregressive moving-average (ARMA) model that we term the time-frequency autoregressive moving-average (TFARMA) model. This model uses frequency shifts in addition to time shifts (delays) for modeling nonstationary process dynamics. The TFARMA model and its special cases, the TFAR and TFMA models, are shown to be specific types of time-varying ARMA (AR, MA) models. They are attractive because of their parsimony for underspread processes, that is, nonstationary processes with a limited time-frequency correlation structure. We develop computationally efficient order-recursive estimators for the TFARMA, TFAR, and TFMA model parameters which are based on linear time-frequency Yule-Walker equations or on a new time-frequency cepstrum. Simulation results demonstrate that the proposed parameter estimators outperform existing estimators for time-varying ARMA (AR, MA) models with respect to accuracy and/or numerical efficiency. An application to the time-varying spectral analysis of a natural signal is also discussed.
\end{abstract}

Index Terms-Cepstrum, nonstationary processes, parametric modeling, time-frequency analysis, time-varying ARMA (TVARMA) models, time-varying spectral estimation, timevarying systems, TVARMA, Yule-Walker equations.

\section{INTRODUCTION}

$\mathbf{N}$ ONSTATIONARY random processes provide an appropriate mathematical framework for signals arising in speech and audio, communications, image processing, computer vision, biomedical engineering, machine monitoring, and many other application fields. Because the statistics of nonstationary random processes depend on time (or space), they are more difficult to describe than the statistics of stationary

Manuscript received March 13, 2006; revised December 25, 2006. The associate editor coordinating the review of this manuscript and approving it for publication was Dr. A. Rahim Leyman. Parts of this work have been previously presented in the Proceedings of the IEEE International Conference on Acoustics, Speech and Signal Processing, Hong Kong, vol. VI, April 2003, pp. 125-128; the Proceedings of the IEEE International Conference on Acoustics, Speech and Signal Processing, Montreal, QC, Canada, vol. II, May 2004, pp. 757-760; the Proceedings of the IEEE International Conference on Acoustics, Speech and Signal Processing, Philadelphia, PA, vol. IV, March 2005, pp. 301-304; and the Proceedings of the IEEE International Workshop on Statistical Signal Processing, Bordeaux, France, July 2005, pp. 909-914.

M. Jachan is with the Freiburg Center for Data Analysis and Modeling, University Medical Center Freiburg, D-79104 Freiburg i. Br., Germany (e-mail: michael.jachan@fdm.uni-freiburg.de).

G. Matz and F. Hlawatsch are with the Institute of Communications and Radio-Frequency Engineering, Vienna University of Technology, A-1040 Vienna, Austria (e-mail: gmatz@nt.tuwien.ac.at; franz.hlawatsch@nt.tuwien.ac. at).

Digital Object Identifier 10.1109/TSP.2007.896265 processes. A parametric second-order description that is parsimonious in that it captures the time-varying second-order statistics by a small number of parameters is hence of particular interest. Here, we propose the use of frequency shifts in addition to time shifts (delays) for modeling nonstationary process dynamics in a physically intuitive way. The resulting parametric models are shown to be equivalent to specific types of time-varying ARMA (TVAR, TVMA) models. They are parsimonious for nonstationary processes with small high-lag temporal and spectral correlations (underspread processes), which are frequently encountered in applications. We also propose efficient order-recursive techniques for model parameter estimation that outperform existing estimators for time-varying ARMA (TVAR, TVMA) models with respect to accuracy and/or complexity.

\section{A. Previous Work}

Time-varying autoregressive moving-average (TVARMA) models generalize the successful time-invariant ARMA models [5], [6] to nonstationary environments [7]-[12]. Consider a zero-mean nonstationary process $x[n]$ defined for $n=0,1, \ldots, N-1$. The TVARMA model of AR order $M_{\mathrm{A}}$ and MA order $M_{\mathrm{B}}$ is given by

$$
\begin{array}{r}
x[n]=-\sum_{m=1}^{M_{\mathrm{A}}} a_{m}[n] x[n-m]+\sum_{\substack{m=0 \\
n=0,1, \ldots, N-1}}^{M_{\mathrm{B}}} b_{m}[n] e[n-m], \\
\text { ( }
\end{array}
$$

where $a_{m}[n]$ and $b_{m}[n]$ are the time-varying parameters of the TVAR and TVMA part, respectively, and $e[n]$ is stationary white noise with variance 1 (the innovations process).

The TVMA and TVAR models are obtained as special cases for $M_{\mathrm{A}}=0$ and $M_{\mathrm{B}}=0$, respectively.

The TVARMA model uses different AR model parameters $a_{m}[n]$ and different MA model parameters $b_{m}[n]$ at each time instant $n$, and thus the total number of model parameters is as high as $N\left(M_{\mathrm{A}}+M_{\mathrm{B}}+1\right)$. Much better parsimony can be achieved by imposing a finite-order basis expansion of the parameter functions, i.e.,

$$
\begin{aligned}
a_{m}[n]=\sum_{l=0}^{L_{\mathrm{A}}} a_{m, l} f_{l}[n], \quad b_{m}[n] & =\sum_{l=0}^{L_{\mathrm{B}}} b_{m, l} f_{l}[n], \\
n & =0,1, \ldots, N-1
\end{aligned}
$$

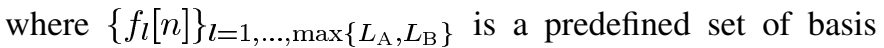
functions [7]-[9], [13]-[19]. The time-varying parameter functions $a_{m}[n], b_{m}[n]$ are described by $M_{\mathrm{A}}\left(L_{\mathrm{A}}+1\right)+$ 
$\left(M_{\mathrm{B}}+1\right)\left(L_{\mathrm{B}}+1\right)$ expansion coefficients $a_{m, l}, b_{m, l}$ that do not depend on time. We will term the resulting TVARMA model a B-TVARMA model. The basis expansion restricts the temporal evolution of $a_{m}[n], b_{m}[n]$ by a subspace constraint. Various types of basis functions have been used, such as polynomials [9], complex exponentials [7], [15], [18], and cosine functions [20].

A central problem is the estimation of the (B-)TVARMA model parameters $a_{m}[n], b_{m}[n]$ or $a_{m, l}, b_{m, l}$ from one or several realizations of the process $x[n]$. For B-TVAR models, an estimator based on vector-Yule-Walker equations was proposed in [8]; its complexity is $\mathcal{O}\left(M_{\mathrm{A}}^{2} L_{\mathrm{A}}^{3}\right)$ [15]. The vector-Yule-Walker equations involve quadratic terms of the basis functions $f_{l}[n]$ and do not generally admit an easy interpretation in terms of the signal statistics. Also, estimation of the time-varying innovations variance $b_{0}^{2}[n]$ is problematic [21]. For B-TVARMA models, the B-TVAR part can be estimated by extended vector-Yule-Walker methods; the B-TVMA part can then be estimated by fitting a long intermediate B-TVAR model and performing an inverse filtering to estimate the innovations process $e[n]$ and turn the nonlinear problem into a linear one [7]. A method for simultaneous estimation of the B-TVMA and B-TVAR parts (without inverse filtering) has been proposed in [21]; this method has been adapted to time-frequency ARMA parameter estimation in [4]. Subspace methods for B-TVMA estimation use a numerically costly eigenvalue or singular value decomposition of a matrix of size $M_{\mathrm{B}} L_{\mathrm{B}} \times M_{\mathrm{B}} L_{\mathrm{B}}$ [20], [22]. B-TVMA models have been applied to time-varying channel modeling, estimation, and equalization [22]-[26]. Maximum-likelihood, lattice, and Schur decomposition methods for B-TVMA estimation [14] are also quite complex. Cepstral methods for TVARMA estimation have been discussed in [27].

\section{B. Main Contributions and Structure of This Paper}

In this paper, we consider a special class of TVARMA models that we term time-frequency ARMA (TFARMA) models. Extending time-invariant ARMA models, which capture temporal dynamics and correlations by representing a process as a weighted sum of time-shifted (delayed) signal components, TFARMA models additionally use frequency shifts to capture a process' nonstationarity and spectral correlations. The lags of the time-frequency (TF) shifts used in the TFARMA model are assumed to be small. This results in nonstationary processes with small high-lag temporal and spectral correlations or, equivalently, with a temporal correlation length that is much smaller than the duration over which the time-varying second-order statistics are approximately constant. Such underspread processes [28], [29] are encountered in many applications.

We will demonstrate that TFARMA models are a TF-symmetric reformulation of B-TVARMA models using a Fourier (complex exponential) basis [7], [15]. The underspread assumption used in this paper results in parsimony, and it will be shown to allow an "underspread approximation" that leads to new, computationally efficient parameter estimators. We present two types of TFAR and TFMA estimators-based on linear TF Yule-Walker equations and on a new TF cepstrum - and we show how these estimators can be combined to obtain TFARMA parameter estimators. In particular, TFAR parameter estimation can be accomplished via underspread $T F$ Yule-Walker equations with Toeplitz/block-Toeplitz structure that can be solved efficiently by means of the Wax-Kailath algorithm [30]. Simulation results demonstrate that our methods outperform existing TVAR, TVMA, and TVARMA parameter estimators with respect to accuracy and/or complexity. For processes that are not underspread (called "overspread" [28], [29]), the models discussed here will not be parsimonious and those estimators that involve an underspread approximation must be expected to exhibit poor performance.

TFARMA models are physically meaningful due to their definition in terms of delays and frequency (Doppler) shifts. This delay-Doppler formulation is also convenient since the nonparametric estimator of the process' second-order statistics that is required for all parametric estimators can be designed and controlled more easily in the delay-Doppler domain. Furthermore, TFARMA models are formulated in a discrete-time, discrete-frequency framework that allows the use of efficient fast Fourier transform (FFT) algorithms. They can be applied in a variety of signal processing tasks, such as time-varying spectral estimation (cf. [17]), time-varying prediction (cf. [1], [7], [15], and [31]), time-varying system approximation [4], prewhitening of nonstationary processes, and nonstationary feature extraction.

This paper is organized as follows. In Section II, we review for later use some TF representations of linear time-varying (LTV) systems and nonstationary random processes. Novel complex TF cepstra (CTFC) of LTV systems and nonstationary random processes are introduced in Section III. In Sections IV and V, we present the TFMA model and the TFAR model, respectively, and we propose associated parameter estimators. In Section VI, we combine the TFMA and TFAR models into the TFARMA model and extend our TFMA and TFAR parameter estimators to the TFARMA case. Sections IV-VI also present simulation results that compare the performance and complexity of the proposed methods with that of existing methods. In Section VII, we apply our models and parameter estimators to the time-varying spectral analysis of a bat echolocation signal.

\section{TIME-FREQUENCY FundAMENTALS}

The basic signal transformations underlying our models are the time shifts $\left(\mathbb{T}^{m} x\right)[n]=x[n-m]$ and frequency shifts (modulations) $\left(\mathbb{M}^{l} x\right)[n]=e^{j 2 \pi l n / N} x[n]$. We assume our signals $x[n]$ to be complex (e.g., the complex representation of a real bandpass signal) and periodic with period $N$, so $\mathbb{T}^{m}$ is actually a cyclic time shift operator (for simplicity of notation, we avoid writing more explicitly $\left.\left(\mathbb{T}^{m} x\right)[n]=x[(n-m) \bmod N]\right)$. The cyclic/periodic time structure is a consequence of our discrete-frequency framework, which allows efficient FFT-based computations. We furthermore combine $\mathbb{T}^{m}$ and $\mathbb{M}^{l}$ into the joint TF shift operator $\mathbb{S}_{m, l} \triangleq \mathbb{M}^{l} \mathbb{T}^{m}$ acting as

$$
\left(\mathbb{S}_{m, l} x\right)[n]=\left(\mathbb{M}^{l} \mathbb{T}^{m} x\right)[n]=e^{j \frac{2 \pi}{N} l n} x[n-m]
$$

where both shift indices $m$ and $l$ are constrained to the range $[-N / 2, N / 2-1]$. 
Next, we discuss some TF representations of LTV systems and nonstationary processes that are based on the TF shift operator $\mathbb{S}_{m, l}$ and will be used later.

\section{A. TF Characterization of LTV Systems}

Consider a causal LTV system $\mathbb{H}$ that operates on discrete-time, finite-length signals $e[n]$ defined on the time interval $[0, N-1]$ according to the input-output relation

$$
\begin{aligned}
x[n] & =(\mathbb{H e})[n] \\
& =\sum_{m=0}^{N / 2-1} h[n, m]\left(\mathbb{T}^{m} e\right)[n] \\
& =\sum_{m=0}^{N / 2-1} h[n, m] e[n-m], \quad n=0,1, \ldots, N-1 .
\end{aligned}
$$

Here, $h[n, m]$ denotes the time-varying impulse response of $\mathbb{H}$ and the summation interval is $[0, N / 2-1]$ due to causality. The

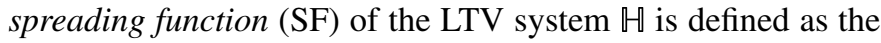
discrete Fourier transform (DFT) of $h[n, m]$ with respect to $n$ [32], [33]:

$$
S_{\mathbb{H}}[m, l] \triangleq \underset{n \rightarrow l}{\mathbb{F}} h[n, m]=\sum_{n=0}^{N-1} h[n, m] e^{-j \frac{2 \pi}{N} l n} .
$$

It is (to within a factor of $1 / N$ ) the coefficient function in an expansion of $\mathbb{H}$ into $\mathrm{TF}$ shift operators $\mathbb{S}_{m, l}$, i.e.,

$$
\mathbb{H}=\frac{1}{N} \sum_{m=0}^{N / 2-1} \sum_{l=-N / 2}^{N / 2-1} S_{\mathbb{H}}[m, l] \mathbb{S}_{m, l} .
$$

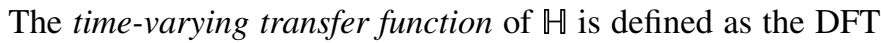
of $h[n, m]$ with respect to $m$ [33], [34]:

$$
Z_{\mathbb{G}}[n, k] \triangleq \underset{m \rightarrow k}{\mathbb{F}} h[n, m]=\sum_{m=0}^{N / 2-1} h[n, m] e^{-j \frac{2 \pi}{N} k m} .
$$

It is the symplectic two-dimensional (2-D) DFT of the SF, i.e.,

$$
\begin{aligned}
Z_{\mathbb{H}}[n, k] & =\underset{m \rightarrow k}{\mathbb{F}} \underset{l \rightarrow n}{\mathbb{F}^{-1}} S_{\mathbb{H}}[m, l] \\
& =\frac{1}{N} \sum_{m=0}^{N / 2-1} \sum_{l=-N / 2}^{N / 2-1} S_{\mathbb{-}[}[m, l] e^{-j \frac{2 \pi}{N}(k m-n l)} .
\end{aligned}
$$

An LTV system is said to be underspread if it introduces TF shifts with only small TF lags [33], [35], [36]. From (4), it follows that the SF of an underspread system is effectively zero outside a small region about the origin of the delay-Doppler plane $((m, l)$ plane). Furthermore, it follows from $(5)$ that the time-varying transfer function of an underspread system is a smooth (lowpass) function.

\section{B. TF Characterization of Nonstationary Random Processes}

Next, consider a nonstationary random process $x[n]$ with correlation function $r_{x}[n, m] \triangleq \mathcal{E}\left\{x[n] x^{*}[n-m]\right\}$, where $\mathcal{E}\{\cdot\}$ denotes expectation (ensemble average). The expected ambiguity function (EAF) of the process $x[n]$, denoted $A_{x}[m, l]$, is defined as [28], [29]

$$
A_{x}[m, l] \triangleq \mathcal{E}\left\{\left\langle x, \mathbb{S}_{m, l} x\right\rangle\right\}=\underset{n \rightarrow l}{\mathbb{F}} r_{x}[n, m]
$$

where $\langle x, y\rangle \triangleq \sum_{n=0}^{N-1} x[n] y^{*}[n]$ is the inner product of two signals $x[n]$ and $y[n]$. Comparison with (3) shows that the EAF is the SF of the correlation operator $\mathbb{R}_{x}$, which is the operator whose kernel (impulse response) is the correlation $r_{x}[n, m]$. The value of $A_{x}[m, l]$ at a given TF lag point $(m, l)$ characterizes the average statistical correlations of any two components of $x[n]$ separated by time lag $m$ and frequency lag $l$. A nonstationary process $x[n]$ is said to be underspread if its TF correlations are effectively zero for larger time and frequency lags [28], [29]. The EAF then is effectively zero outside a small region about the origin of the $(m, l)$ plane.

A nonstationary process can be represented as the output of a causal LTV system $\mathbb{H}_{x}$ (an innovations system) whose input is stationary white noise $e[n]$ with unit variance (the innovations process) [37], i.e.,

$$
\begin{array}{rl}
x[n]=\left(\mathbb{H}_{x} e\right)[n]=\sum_{m=0}^{N / 2-1} h_{x}[n, m] e[n-m], & \\
n & n=0,1, \ldots, N-1 .
\end{array}
$$

By inserting (7) into (6) and evaluating the expectation, the EAF of $x[n]$ is expressed as

$$
\begin{aligned}
A_{x}[m, l]=\frac{1}{N} \sum_{m^{\prime}=0}^{N / 2-1} & \sum_{l^{\prime}=-N / 2}^{N / 2-1} S_{\mathbb{H}_{x}}\left[m^{\prime}, l^{\prime}\right] \\
& \times S_{\mathbb{H}_{x}}^{*}\left[m^{\prime}-m, l^{\prime}-l\right] e^{-j \frac{2 \pi}{N} m\left(l-l^{\prime}\right)} .
\end{aligned}
$$

The evolutionary spectrum is defined as [38], [39]

$$
G_{x}[n, k] \triangleq\left|Z_{\mathbb{H}_{x}}[n, k]\right|^{2} .
$$

If $\mathrm{H}_{x}$ is underspread in the sense of Section II-A, then $x[n]$ is underspread as well, and the evolutionary spectrum can be approximated by the 2-D DFT of the EAF [28], [29], i.e.,

$$
G_{x}[n, k] \approx \underset{m \rightarrow k}{\mathbb{F}} \underset{l \rightarrow n}{\mathbb{F}^{-1}} A_{x}[m, l] .
$$

Conversely, if $x[n]$ is underspread, one can always find an underspread innovations system $\mathbb{H}_{x}$. Let us assume that $\mathbb{H}_{x}$ is underspread in the sense that the SF $S_{\mathbb{H}_{x}}[m, l]$ is effectively zero outside the rectangle $[0, M] \times[-L, L]$ with $M L \ll N$. Due to (8), the EAF will then be effectively zero outside the extended rectangle $[-M, M] \times[-2 L, 2 L]$. Furthermore, in the EAF expression (8), the phase factor $e^{-j 2 \pi m\left(l-l^{\prime}\right) / N}$ can then be approximated by 1 because

$$
\begin{aligned}
& \max _{\substack{m \in[-M, M] \\
l-l^{\prime} \in[-2 L, 2 L]}}\left|e^{-j \frac{2 \pi}{N} m\left(l-l^{\prime}\right)}-1\right| \\
& \quad=2\left|\sin \left(\frac{\pi M \cdot 2 L}{N}\right)\right| \leq 4 \pi \frac{M L}{N} \ll 1 .
\end{aligned}
$$


Hence, the EAF of the underspread process $x[n]$ is approximated as

$$
\begin{aligned}
A_{x}[m, l] & \approx A_{x}^{(u)}[m, l] \\
& \triangleq \frac{1}{N} \sum_{m^{\prime}=0}^{M} \sum_{l^{\prime}=-L}^{L} S_{\mathbb{H}_{x}}\left[m^{\prime}, l^{\prime}\right] S_{\uplus_{x}}^{*}\left[m^{\prime}-m, l^{\prime}-l\right]
\end{aligned}
$$

which is the cyclic 2-D autocorrelation of $S_{\uplus_{x}}[m, l]$.

\section{COMPLEX Time-Frequency CePSTRA}

The cepstrum of a linear time-invariant system can be used to develop a nonlinear parameter estimator for time-invariant ARMA models [40]. The cepstral method has been generalized to LTV systems and TV(AR)MA models in [27]. In this section, we introduce novel TF cepstra that will be used later to develop TFMA and TFARMA parameter estimators that improve on the methods of [27] in terms of accuracy and complexity [2].

\section{A. Complex TF Cepstrum of LTV Systems}

Consider a causal and minimum-phase ${ }^{1}$ LTV system $\mathbb{H}$. We define a time-varying transfer function of $\mathbb{H}$ in the complex $(u, z)$-biplane as the following 2-D $z$-transform of the SF:

$$
Z_{\mathbb{H}}(u, z) \triangleq \frac{1}{N} \sum_{m=0}^{N / 2-1} \sum_{l=-N / 2}^{N / 2-1} S_{\mathbb{-}}[m, l] u^{l} z^{-m} .
$$

The function $Z_{\mathbb{H}}(u, z)$ is analytic for all $u, z$ because of the finite summation limits. We assume that $Z_{\mathbb{H}}(u, z) \neq 0$ in a region $\mathcal{R}$ defined by $1-\epsilon \leq|u| \leq 1+\epsilon$ and $1-\epsilon \leq|z| \leq 1+\epsilon$ with some positive constant $\epsilon$; this guarantees that $\log \left(Z_{\mathbb{H}}(u, z)\right)$ is analytic in $\mathcal{R}$. Note that the time-varying transfer function $Z_{\mathbb{H}}[n, k]$ in (5) is reobtained by sampling $Z_{\sharp \sharp}(u, z)$ on the unit bicircle, i.e., $Z_{\mathbb{H}}[n, k]=Z_{\mathbb{H}}\left(e^{j 2 \pi n / N}, e^{j 2 \pi k / N}\right)$.

The complex TF cepstrum (CTFC) of the causal, minimumphase LTV system $\mathbb{H}$, denoted as $^{2} \widetilde{\mathfrak{S}}_{\mathbb{H}}[m, l]$, is now defined implicitly by setting

$$
\log \left(Z_{\mathbb{H}}(u, z)\right)=\frac{1}{N} \sum_{m=-\infty}^{\infty} \sum_{l=-\infty}^{\infty} \tilde{\mathfrak{S}}_{\mathbb{H}}[m, l] u^{l} z^{-m} .
$$

It can be shown that $\widetilde{\mathfrak{S}}_{\mathbb{H}}[m, l]=0$ for $m<0$ because $\mathbb{H}$ is minimum-phase, and that $\widetilde{\mathfrak{S}}_{\mathbb{H}}[m, l]$ has infinite length with respect to both $m$ and $l$ but decays at least as $1 / m$ and $1 / l$ (cf. [41] for the case of a time-invariant system).

For an approximate computation of the CTFC using DFTs, we sample (13) on the unit bicircle, i.e., we set $u=e^{j 2 \pi n / N}$ and $z=e^{j 2 \pi k / N}$. We obtain

$$
\begin{aligned}
\log \left(Z_{\mathbb{H}}[n, k]\right) & =\frac{1}{N} \sum_{m=0}^{\infty} \sum_{l=-\infty}^{\infty} \tilde{\mathfrak{S}}_{\mathbb{H}}[m, l] e^{-j \frac{2 \pi}{N}(k m-n l)} \\
& =\underset{m \rightarrow k}{\mathbb{F}} \underset{l \rightarrow n}{\mathbb{F}^{-1} \mathfrak{S}_{\mathbb{H}}[m, l]}
\end{aligned}
$$

\footnotetext{
${ }^{1}$ An LTV system will be termed minimum-phase if its SF $S_{H}[m, l]$ is a minimum-phase (and, thus, causal) sequence in $m$ for each $l$.

${ }^{2}$ In what follows, we will use the tilde $\tilde{*}$ to indicate that a function is not $N$-periodic.
}

where $\mathfrak{S}_{\mathbb{H}}[\mathrm{m}, l]$, termed the cyclic $C T F C$, is a periodized version of the CTFC, i.e.,

$$
\mathfrak{S}_{\mathbb{H}}[m, l] \triangleq \sum_{i=-\infty}^{\infty} \sum_{i^{\prime}=-\infty}^{\infty} \tilde{\mathfrak{S}}_{\mathbb{H}}\left[m+i N, l+i^{\prime} N\right] .
$$

By inserting (5) into (14) and inverting the DFTs, we see that the cyclic CTFC can be calculated as

$$
\mathfrak{S}_{\mathbb{H}}[m, l]=\underset{k \rightarrow m}{\mathbb{F}} \mathbb{n}_{n \rightarrow l}^{-1} \mathbb{F} \log \left(\underset{m^{\prime} \rightarrow k}{\mathbb{F}} \underset{l^{\prime} \rightarrow n}{\mathbb{F}^{-1}} S_{\mathbb{H}}\left[m^{\prime}, l^{\prime}\right]\right) .
$$

If $\tilde{\mathfrak{S}}_{\mathbb{H}}[m, l]$ is sufficiently decayed for $(m, l) \notin[-N / 2, N / 2-$ 1] $\times[-N / 2, N / 2-1]$, the cyclic CTFC is effectively equal to the CTFC, i.e., $\mathfrak{S}_{\mathbb{H}}[m, l] \approx \tilde{\mathfrak{S}}_{\mathbb{H}}[m, l]$ for $(m, l) \in[-N / 2, N / 2-$ $1] \times[-N / 2, N / 2-1]$.

\section{B. Complex TF Cepstrum of Nonstationary Processes}

In an analogy to (14), we define the cyclic CTFC of a nonstationary process $x[n]$, denoted as $\mathfrak{A}_{x}[m, l]$, by

$$
\log \left(G_{x}[n, k]\right)=\underset{m \rightarrow k}{\mathbb{F}} \underset{l \rightarrow n}{\mathbb{F}} \mathbb{F}^{-1} \mathfrak{A}_{x}[m, l]
$$

[cf. (9) and (10)]. By inserting (10) into (17) and inverting the DFTs, it is seen that for an underspread process, $\mathfrak{A}_{x}[m, l]$ can be calculated from the EAF $A_{x}[m, l]$ by an expression that is analogous to (16):

$$
\mathfrak{A}_{x}[m, l] \approx \underset{k \rightarrow m}{\mathbb{F}} \mathbb{F}^{-1} \underset{n \rightarrow l}{\mathbb{F}} \log \left(\underset{m^{\prime} \rightarrow k}{\mathbb{F}} \underset{l^{\prime} \rightarrow n}{\mathbb{F}^{-1}} A_{x}\left[m^{\prime}, l^{\prime}\right]\right) .
$$

Taking the logarithm of (9), we obtain $\log \left(G_{x}[n, k]\right)=$ $\log \left(Z_{\mathbb{H}_{x}}[n, k]\right)+\log \left(Z_{\mathbb{H}_{x}}^{*}[n, k]\right)$, which implies via (17) and (14)

$$
\mathfrak{A}_{x}[m, l]=\mathfrak{S}_{\mathbb{H}_{x}}[m, l]+\mathfrak{S}_{\mathbb{H}_{x}}^{*}[-m,-l] .
$$

This relation expresses the cyclic CTFC of the process $x[n]$ in terms of the cyclic CTFC of the innovations system $\mathbb{H}_{x}$ (cf. [41] for the case of a time-invariant system). Since $\mathbb{H}_{x}$ was assumed to be a minimum-phase system, $\mathfrak{S}_{\mathbb{H}_{x}}[m, l]$ vanishes for $m<0$ and thus (19) implies

$$
\mathfrak{A}_{x}[m, l]= \begin{cases}\mathfrak{S}_{\mathbb{H}_{x}}[m, l], & m>0 \\ 2 \mathfrak{S}_{\mathbb{H}_{x}}[0, l], & m=0 \\ \mathfrak{S}_{\mathbb{H}_{x}}^{*}[-m,-l], & m<0 .\end{cases}
$$

\section{TFMA Modeling AND PARAMETER Estimation}

In this and the next two sections, we present three different parametric models and corresponding parameter estimation methods for length- $N$ nonstationary random processes $x[n]$, $n=0,1, \ldots, N-1$. These models are all based on the TF shift operator $\mathbb{S}_{m, l}$. We first discuss the TF moving average (TFMA) model. TFMA models are especially suited for processes whose spectra exhibit deep time-varying nulls but no spectral peaks. An example of a signal that is well suited to a representation by a TFMA model is shown in Fig. 1. 

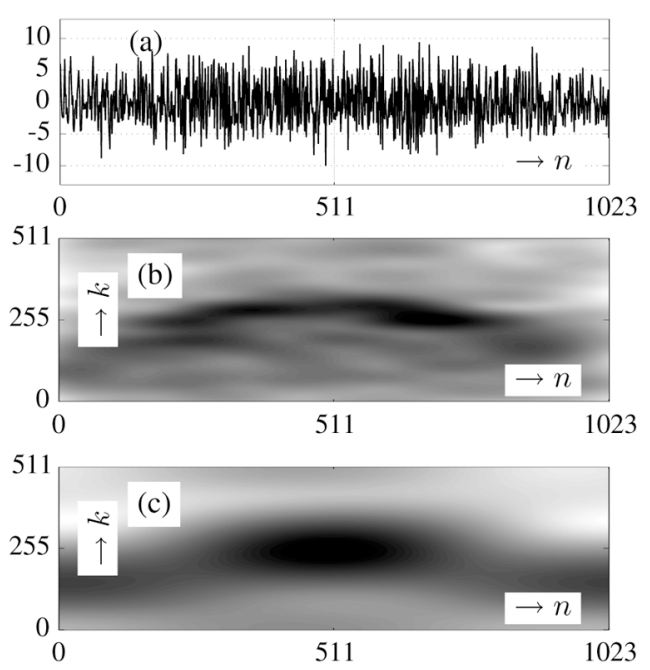

Fig. 1. Spectral analysis of a blood pressure signal (http://spib.rice.edu): (a) time-domain signal, (b) smoothed pseudo-Wigner distribution [42], [43], and (c) $\operatorname{TFMA}(4,1)$ spectral estimate as defined by $(25)$. Logarithmic gray-scale representations are used in (b) and (c).

\section{A. TFMA Model}

We define the TFMA $\left(M_{\mathrm{B}}, L_{\mathrm{B}}\right)$ model [2] by the input-output relation

$$
\begin{aligned}
x[n] & \triangleq \sum_{m=0}^{M_{\mathrm{B}}} \sum_{l=-L_{\mathrm{B}}}^{L_{\mathrm{B}}} b_{m, l}\left(\mathbb{S}_{m, l} e\right)[n] \\
& =\sum_{m=0}^{M_{\mathrm{B}}} \sum_{l=-L_{\mathrm{B}}}^{L_{\mathrm{B}}} b_{m, l} e^{j \frac{2 \pi}{N} l n} e[n-m], \quad n=0,1, \ldots, N-1 .
\end{aligned}
$$

Here, the innovations process $e[n]$ is stationary white noise with variance $1 ; M_{\mathrm{B}}$ and $L_{\mathrm{B}}$ denote the temporal (delay) and spectral (Doppler) model orders, respectively; and the $N_{\mathbb{B}} \triangleq\left(M_{\mathrm{B}}+1\right)\left(2 L_{\mathrm{B}}+1\right)$ constants $b_{m, l}, m=0, \ldots, M_{\mathrm{B}}, l=$ $-L_{\mathrm{B}}, \ldots, L_{\mathrm{B}}$ are the TFMA parameters. Fig. 2 depicts the TFMA input-output relation (21) in the form $x[n]=$ $\sum_{m=0}^{M_{\mathrm{B}}}\left(\sum_{l=-L_{\mathrm{B}}}^{L_{\mathrm{B}}} b_{m, l} e^{j 2 \pi l n / N}\right) e[n-m]$. This is a generalized tapped delay line where the taps are in fact modulation circuits. A different though mathematically equivalent block diagram can be obtained by writing (21) as $x[n]=$ $\sum_{l=-L_{\mathrm{B}}}^{L_{\mathrm{B}}}\left(\sum_{m=0}^{M_{\mathrm{B}}} b_{m, l} e[n-m]\right) e^{j 2 \pi l n / N}$. For $L_{\mathrm{B}}=0$, a cyclic version of the classical time-invariant MA model [5], [6] is obtained as a special case of the TFMA model.

According to its definition in (21), the $\operatorname{TFMA}\left(M_{\mathrm{B}}, L_{\mathrm{B}}\right)$ process $x[n]$ is modeled as a linear combination of TF shifted versions of the white noise $e[n]$. This is a special case of the innovations system representation $x[n]=\left(\mathbb{H}_{x} e\right)[n]$ in (7); the innovations system $\mathbb{H}_{x}$ is given by the causal, nonrecursive LTV system

$$
\mathbb{B} \triangleq \sum_{m=0}^{M_{\mathrm{B}}} \sum_{l=-L_{\mathrm{B}}}^{L_{\mathrm{B}}} b_{m, l} \mathbb{S}_{m, l}
$$

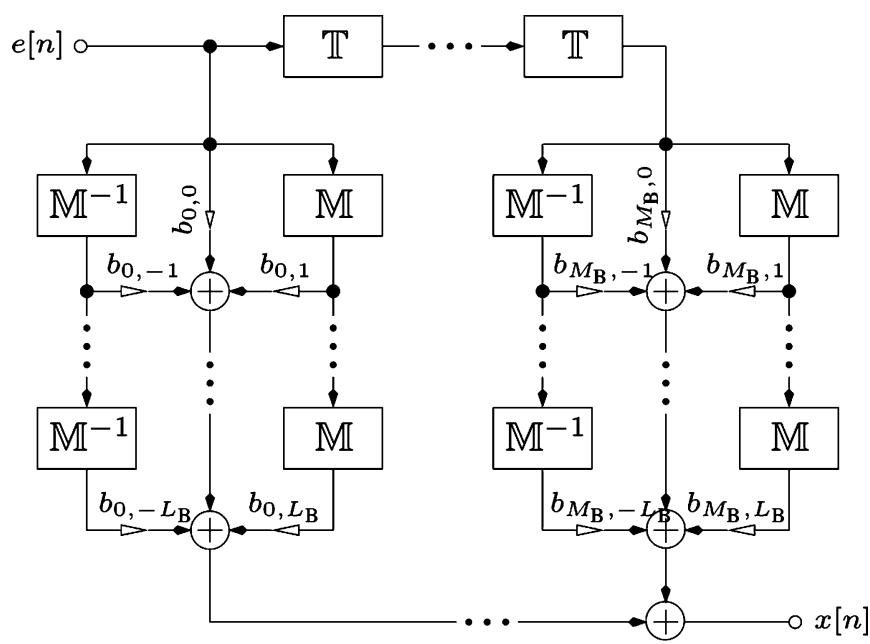

Fig. 2. Block diagram of the TFMA $\left(M_{\mathrm{B}}, L_{\mathrm{B}}\right)$ model. Unfilled arrows denote multiplication by constants (model parameters $b_{m, l}$ ).

Comparing with (4), we see that the SF of $\mathbb{B}$ is given by

$$
S_{\mathbb{B}}[m, l]= \begin{cases}N b_{m, l}, & (m, l) \in \mathcal{B} \\ 0, & \text { elsewhere }\end{cases}
$$

where $\mathcal{B} \triangleq\left[0, M_{\mathrm{B}}\right] \times\left[-L_{\mathrm{B}}, L_{\mathrm{B}}\right]$. That is, the $\mathrm{SF}$ of $\mathbb{B}$ is zero outside a rectangle about the origin, and the nonzero SF values are (up to a factor) the TFMA parameters $b_{m, l}$. For later convenience, we define $b_{m, l} \triangleq 0$ for $(m, l) \notin \mathcal{B}$.

The TFMA model is easily seen to be a special case of the B-TVMA model. Indeed, it is a special case of (1) and (2) with $M_{\mathrm{A}}=0$ and the Fourier (complex exponential) basis $\left\{f_{l}[n]=\right.$ $\left.e^{j 2 \pi l n / N}\right\}_{l=-L_{\mathrm{B}}, \ldots, L_{\mathrm{B}}}[7]$. The time-varying MA parameters in (1), (2) are given by

$$
b_{m}[n]=\sum_{l=-L_{\mathrm{B}}}^{L_{\mathrm{B}}} b_{m, l} e^{j \frac{2 \pi}{N} l n} .
$$

This means that the functions $b_{m}[n]$ are band-limited with bandwidth $L_{\mathrm{B}}$, resulting in a time variation that is smooth but may still be rapid for sufficiently large Doppler order $L_{\mathrm{B}}$. The Fourier basis has the advantage that inner products can be computed by FFT methods with a complexity of $\mathcal{O}(N \log N)$ (instead of $\mathcal{O}\left(N^{2}\right)$ ). The zero-delay parameter $b_{0}[n]$ can be interpreted as a time-varying standard deviation of the innovations and is hence constrained to be positive for all $n$; this means that $b_{0, l}$ is an autocorrelation function in $l$. Our formulation in terms of TF shifts provides a new interpretation of B-TVMA models, and it suggests the application of TF signal representations and $\mathrm{TF}$ concepts ( $\mathrm{SF}$, time-varying transfer function, EAF, underspread systems and processes) for efficient estimation of the TFMA parameters $b_{m, l}$. This will be worked out in the rest of this section.

The EAF of a TFMA $\left(M_{\mathrm{B}}, L_{\mathrm{B}}\right)$ process $x[n]$ is obtained from (21) and (6) as

$$
A_{x}[m, l]=N \sum_{m^{\prime}=0}^{M_{\mathrm{B}}} \sum_{l^{\prime}=-L_{\mathrm{B}}}^{L_{\mathrm{B}}} b_{m^{\prime}, l^{\prime}} b_{m^{\prime}-m, l^{\prime}-l}^{*} e^{-j \frac{2 \pi}{N} m\left(l-l^{\prime}\right)} .
$$


Note that there are temporal correlations up to time lag $\pm M_{\mathrm{B}}$ and spectral correlations up to frequency lag $\pm 2 L_{\mathrm{B}}$. The evolutionary spectrum (9) is given by (recall that $\mathbb{H}_{x}=\mathbb{B}$ )

$$
G_{x}[n, k]=\left|Z_{\mathbb{B}}[n, k]\right|^{2}=\left|\sum_{m=0}^{M_{\mathrm{B}}} \sum_{l=-L_{\mathrm{B}}}^{L_{\mathrm{B}}} b_{m, l} e^{j \frac{2 \pi}{N}(n l-k m)}\right|^{2} .
$$

\section{B. TFMA Parameter Estimation Based on the CTFC Recursion}

We now present a nonlinear method for estimating the TFMA parameters $b_{m, l}$ from a realization of the process $x[n]$. This method is based on the CTFC introduced in Section III and assumes a minimum-phase innovations system $\mathbb{B}$. Following the approach for time-invariant ARMA estimation in [40], we first derive a cepstral recursion for the $b_{m, l}$ 's that involves the cyclic CTFC $\mathfrak{S}_{\mathbb{B}}[m, l]$. Subsequently, we express this recursion in terms of an estimate of the cyclic CTFC $\mathfrak{A}_{x}[m, l]$, in which form it can be used for TFMA parameter estimation. The resulting estimator is more accurate and less complex than the method proposed in [27], due to the smaller number of parameters to be estimated (see Section IV-D). An alternative TFMA parameter estimator involving a high-order intermediate TFAR model will be presented in Section IV-C.

1) CTFC Recursion: We start by specializing the CTFC definition (13) to the TFMA system $\mathbb{B}$ in (22), as follows:

$\log \left(\sum_{m=0}^{M_{\mathrm{B}}} \sum_{l=-L_{\mathrm{B}}}^{L_{\mathrm{B}}} b_{m, l} u^{l} z^{-m}\right)=\frac{1}{N} \sum_{m=0}^{\infty} \sum_{l=-\infty}^{\infty} \tilde{\mathfrak{S}}_{\mathbb{B}}[m, l] u^{l} z^{-m}$

where (12) and (23) have been used. Differentiating this equation with respect to $z^{-1}$ yields

$$
\begin{aligned}
\sum_{m=1}^{M_{\mathrm{B}}} & \sum_{l=-L_{\mathrm{B}}}^{L_{\mathrm{B}}} m b_{m, l} u^{l} z^{-m+1} \\
= & \frac{1}{N} \sum_{m^{\prime \prime}=1}^{\infty} \sum_{l^{\prime \prime}=-\infty}^{\infty} m^{\prime \prime} \tilde{\mathfrak{S}}_{\mathbb{B}}\left[m^{\prime \prime}, l^{\prime \prime}\right] \\
& \times \sum_{m^{\prime}=0}^{M_{\mathrm{B}}} \sum_{l^{\prime}=-L_{\mathrm{B}}}^{L_{\mathrm{B}}} b_{m^{\prime}, l^{\prime}} u^{l^{\prime}+l^{\prime \prime}} z^{-m^{\prime}-m^{\prime \prime}+1} .
\end{aligned}
$$

Using the index transformations $l^{\prime \prime}=l-l^{\prime}$ and $m^{\prime \prime}=m-m^{\prime}$ on the right-hand side, approximating $\widetilde{\mathfrak{S}}_{\mathbb{B}}[m, l]$ by the cyclic CTFC $\mathfrak{S}_{\mathbb{B}}[m, l]$ [cf. (15)], matching coefficients in $u^{l}$ and $z^{-m}$, and using the fact that $\mathfrak{S}_{\mathbb{B}}[m, l]=0$ for $-N / 2 \leq m<0$, we obtain the CTFC recursion

$$
\begin{gathered}
b_{m, l} \approx \frac{1}{N} \sum_{m^{\prime}=0}^{m-1} \sum_{l^{\prime}=-L_{\mathrm{B}}}^{L_{\mathrm{B}}} \frac{m-m^{\prime}}{m} b_{m^{\prime}, l^{\prime}} \mathfrak{S}_{\mathbb{B}}\left[m-m^{\prime}, l-l^{\prime}\right], \\
m=1, \ldots, M_{\mathrm{B}}, l=-L_{\mathrm{B}}, \ldots, L_{\mathrm{B}} .
\end{gathered}
$$

Later, we will express this recursion in terms of $\mathfrak{A}_{x}[m, l]$.

2) Initialization: The above recursion allows an $m$-recursive calculation of the TFMA parameters $b_{m, l}$. It is initialized by $b_{0, l}$, which can be calculated as follows. The time-varying MA parameters $b_{m}[n]=\sum_{l=-L_{\mathrm{B}}}^{L_{\mathrm{B}}} b_{m, l} e^{j \frac{2 \pi}{N} n l}$ in (24) can be factored as $b_{m}[n]=b_{0}[n] b_{m}^{\prime}[n]$, where $b_{0}[n]>0$ is a time-varying amplitude and $b_{0}^{\prime}[n]=1$ (i.e., $b_{m}^{\prime}[n]$ corresponds to a monic system $\left.\mathbb{B}^{\prime}\right)$. We then obtain for the time-varying transfer function $Z_{\mathbb{B}}[n, k]=b_{0}[n] Z_{\mathbb{B}^{\prime}}[n, k]$, with $Z_{\mathbb{B}^{\prime}}[n, k]=\sum_{m=0}^{M_{\mathrm{B}}} b_{m}^{\prime}[n] e^{-j 2 \pi k m / N}$. Taking the logarithm yields $\log \left(Z_{\mathbb{B}}[n, k]\right)=\log \left(b_{0}[n]\right)+\log \left(Z_{\mathbb{B}^{\prime}}[n, k]\right)$. Inserting this relation into (14), we obtain the cyclic CTFC as

$$
\mathfrak{S}_{\mathbb{B}}[m, l]=\delta[m] \underset{n \rightarrow l}{\mathbb{F}} \log \left(b_{0}[n]\right)+\mathfrak{S}_{\mathbb{B}^{\prime}}[m, l] .
$$

Because $\mathbb{B}$ was assumed minimum phase, $\mathbb{B}^{\prime}$ is minimum phase as well. It can be shown that the CTFC of a monic minimumphase LTV system vanishes for $m=0$, and thus $\mathfrak{S}_{\mathbb{B}^{\prime}}[0, l] \equiv 0$ (cf. [41] for the case of a time-invariant system). Hence, (27) evaluated for $m=0$ becomes

$$
\mathfrak{S}_{\mathbb{B}}[0, l]=\underset{n \rightarrow l}{\mathbb{F}} \log \left(b_{0}[n]\right)=\underset{n \rightarrow l}{\mathbb{F}} \log \left(N \underset{l^{\prime} \rightarrow n}{\mathbb{F}}{ }^{-1} b_{0, l^{\prime}}\right) .
$$

Solving for $b_{0, l}$ finally yields the desired initialization of (26) as

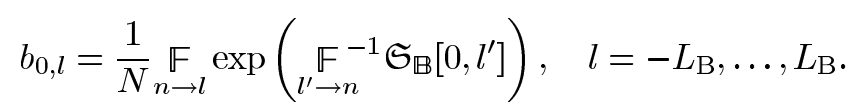

3) TFMA Parameter Estimator: Next, we express the CTFC recursion (26), (28) in terms of the second-order statistics of the TFMA process $x[n]$. Using (20), the CTFC recursion (26) can be written in terms of $\mathfrak{A}_{x}[m, l]$ as

$$
\begin{array}{r}
b_{m, l} \approx \frac{1}{N} \sum_{m^{\prime}=0}^{m-1} \sum_{l^{\prime}=-L_{\mathrm{B}}}^{L_{\mathrm{B}}} \frac{m-m^{\prime}}{m} b_{m^{\prime}, l^{\prime}} \mathfrak{A}_{x}\left[m-m^{\prime}, l-l^{\prime}\right], \\
m=1, \ldots, M_{\mathrm{B}}, l=-L_{\mathrm{B}}, \ldots, L_{\mathrm{B}}
\end{array}
$$

which is initialized by [cf. (28)]

$b_{0, l} \approx \frac{1}{N} \underset{n \rightarrow l}{\mathbb{F}} \exp \left(\frac{1}{2} \underset{l^{\prime} \rightarrow n}{\mathbb{F}^{-1}} \mathfrak{A}_{x}\left[0, l^{\prime}\right]\right), \quad l=-L_{\mathrm{B}}, \ldots, L_{\mathrm{B}}$.

A practical estimator for the TFMA parameters $b_{m, l}$ is finally obtained by treating the approximations (29) and (30) as exact equations, and replacing $\mathfrak{A}_{x}[m, l]$ by an estimate that is derived from an estimate of the EAF $A_{x}[m, l]$ according to (18) (note that the latter step is based on the underspread assumption $\left.M_{\mathrm{B}} L_{\mathrm{B}} \ll N\right)$. Using this method, estimates $b_{m, l}$ of the TFMA parameters are calculated recursively in $m$.

The computational complexity of this parameter estimator-not counting estimation of the EAF-is as follows. Calculation of the CTFC estimate from the EAF estimate according to (18) requires $4 N$ FFTs of length $N$ and $N^{2}$ real logarithms. The CTFC recursion (29) (the initialization (30) has negligible complexity) requires $\mathcal{O}\left(M_{\mathrm{B}}^{2} L_{\mathrm{B}}^{2}\right)$ operations (multiplications). Thus, the overall complexity per signal sample is $\mathcal{O}\left(N \log N+M_{\mathrm{B}}^{2} L_{\mathrm{B}}^{2} / N\right)$ operations plus $N$ logarithms. In the underspread case $\left(M_{\mathrm{B}} L_{\mathrm{B}} \ll N\right), M_{\mathrm{B}}^{2} L_{\mathrm{B}}^{2} / N$ is small and our method is less computationally intensive than the cepstral TVMA estimator [27], whose complexity per signal sample is $\mathcal{O}\left(N \log N+M_{\mathrm{B}}^{2}\right)$ operations plus $N$ logarithms. This will be verified experimentally in Section IV-D. 
4) EAF Estimation: The above TFMA parameter estimator uses an estimate of the EAF $A_{x}[m, l]$. A widely used type of EAF estimator is given by [42], [44], [45]

$$
\hat{A}_{x}[m, l]=\chi_{x}[m, l] \Psi[m, l]
$$

where $\chi_{x}[m, l] \triangleq \underset{n \rightarrow l}{\mathbb{F}}\left\{x[n] x^{*}[n-m]\right\}$ is the ambiguity function of the observed signal $x[n]$ (note that $A_{x}[m, l]=\mathcal{E}\left\{\chi_{x}[m, l]\right\}$ ) and $\Psi[m, l]$ is a 2-D taper function that attenuates $\chi_{x}[m, l]$ for larger lags $m, l$. (Here, as always, $x[n] x^{*}[n-m]$ is short for the cyclic definition $x[n] x^{*}[(n-m) \bmod N]$.) The taper function has to satisfy the normalization property $\Psi[0,0]=1$ and the symmetry property $\Psi[m, l]=e^{-j \frac{2 \pi}{N} m l} \Psi^{*}[-m,-l]$ (because the latter property is satisfied by the EAF). For CTFC-based parameter estimators, the TF taper also needs to be positive definite. A method for taper design is presented in [42]. When $I$ observations $x_{i}[n]$ of the process are available, the EAF estimator (31) is modified by replacing $\chi_{x}[m, l]$ with $(1 / I) \sum_{i=1}^{I} \chi_{x_{i}}[m, l]$.

\section{TFMA Parameter Estimation Based on an Intermediate TFAR Model}

A second TFMA parameter estimator uses an intermediate high-order TFAR model and inverse filtering (cf. [7] and [46]-[48] in the time-invariant case). Let us form the inner product of $(21)$ with $\left(\mathbb{S}_{m, l} x\right)[n]$ and take expectations

$$
\mathcal{E}\left\{\left\langle x, \mathbb{S}_{m, l} x\right\rangle\right\}=\sum_{m^{\prime}=0}^{M_{\mathrm{B}}} \sum_{l^{\prime}=-L_{\mathrm{B}}}^{L_{\mathrm{B}}} b_{m^{\prime}, l^{\prime}} \mathcal{E}\left\{\left\langle\mathbb{S}_{m^{\prime}, l^{\prime}} e, \mathbb{S}_{m, l} x\right\rangle\right\} .
$$

The left-hand side is recognized as the EAF $A_{x}[m, l]$ in (6). On the right-hand side, $\mathcal{E}\left\{\left\langle\mathbb{S}_{m^{\prime}, l^{\prime}} e, \mathbb{S}_{m, l} x\right\rangle\right\}=A_{e, x}\left[m-m^{\prime}, l-\right.$ $\left.l^{\prime}\right] e^{-j \frac{2 \pi}{N} m^{\prime}\left(l-l^{\prime}\right)}$ with the cross-EAF $A_{e, x}[m, l] \triangleq \mathcal{E}\left\{\left\langle e, \mathbb{S}_{m, l} x\right\rangle\right\}$. Considering (32) for $m=0, \ldots, M_{\mathrm{B}}$ and $l=-L_{\mathrm{B}}, \ldots, L_{\mathrm{B}}$, we then obtain

$$
\begin{gathered}
\sum_{m^{\prime}=0}^{M_{\mathrm{B}}} \sum_{l^{\prime}=-L_{\mathrm{B}}}^{L_{\mathrm{B}}} b_{m^{\prime}, l^{\prime}} A_{e, x}\left[m-m^{\prime}, l-l^{\prime}\right] e^{-j \frac{2 \pi}{N} m^{\prime}\left(l-l^{\prime}\right)}=A_{x}[m, l], \\
m=0, \ldots, M_{\mathrm{B}}, l=-L_{\mathrm{B}}, \ldots, L_{\mathrm{B}} .
\end{gathered}
$$

These are $N_{\mathbb{B}}=\left(M_{\mathrm{B}}+1\right)\left(2 L_{\mathrm{B}}+1\right)$ linear equations in the $N_{\mathbb{B}}$ unknowns (TFMA parameters) $b_{m, l}, m=0, \ldots, M_{\mathrm{B}}$, $l=-L_{\mathrm{B}}, \ldots, L_{\mathrm{B}}$. The overall estimation method follows a system identification approach because the $b_{m, l}$ 's are estimated from the output $x[n]$ and the (estimated) input $e[n]$ of the innovations system. Indeed, this method can be shown to be essentially equivalent - up to border effects due to our cyclic framework that have little influence on accuracy and complexity-to the B-TVMA estimator of [7].

If $\mathbb{B}$ is underspread, i.e., $M_{\mathrm{B}} L_{\mathrm{B}} \ll N$, we can use a bound similar to (11) to show that the phase factor $e^{-j \frac{2 \pi}{N} m^{\prime}\left(l-l^{\prime}\right)}$ in (33) can be approximated by 1 . Then, (33) simplifies to the 2-D convolution relation

$$
\begin{aligned}
\sum_{m^{\prime}=0}^{M_{\mathrm{B}}} \sum_{l^{\prime}=-L_{\mathrm{B}}}^{L_{\mathrm{B}}} b_{m^{\prime}, l^{\prime}} A_{e, x}\left[m-m^{\prime}, l-l^{\prime}\right] & =A_{x}[m, l], \\
m=0, \ldots, M_{\mathrm{B}}, l & =-L_{\mathrm{B}}, \ldots, L_{\mathrm{B}} .
\end{aligned}
$$

Using suitable stacking, both the exact equations (33) and the underspread approximation (34) can be written in matrix-vector form as $\boldsymbol{C} \boldsymbol{b}=\boldsymbol{c}$, where the length- $N_{\mathbb{B}}$ vector $\boldsymbol{b}$ contains the TFMA parameters $b_{m, l}$ and the $N_{\mathbb{B}} \times N_{\mathbb{B}}$ matrix $C$ and length- $N_{\mathbb{B}}$ vector $c$ contain appropriate samples of $A_{e, x}$ and $A_{x}$, respectively. A specific stacking will be discussed in a different context in Sections V-B and V-C. Using this stacking, the system matrix $C$ corresponding (34) has a Toeplitz/block-Toeplitz (TBT) structure. This allows the use of the Wax-Kailath algorithm [30] for efficient solution of the underspread equations with a complexity of $\mathcal{O}\left(M_{\mathrm{B}}^{2} L_{\mathrm{B}}^{3}\right)$ multiplications. The recursive structure of the Wax-Kailath algorithm results in an orderrecursive estimator (recursive in the delay order $M_{\mathrm{B}}$ ). Thus, we are able to successively estimate all $\operatorname{TFMA}\left(M_{\mathrm{B}}, L_{\mathrm{B}}\right)$ models with $M_{\mathrm{B}}$ ranging from 0 to a prescribed maximum order.

For a practical TFMA estimator, $A_{x}[m, l]$ and $A_{e, x}[m, l]$ are estimated from a process realization $x[n]$ (we note that the EAF estimator (31) can be extended to the cross-EAF). For estimation of $A_{e, x}[m, l]$, we first have to estimate the innovations signal $e[n]=\left(\mathbb{B}^{-1} x\right)[n]$ from $x[n]$. To this end, the TFMA model underlying $x[n]$ is approximated by an intermediate high-order TFAR model, i.e., we fit a TFAR model (see Section V-A) to $x[n]$. The TFAR order can be determined as discussed in [3], and the TFAR parameters can be estimated by means of the methods presented in Section V. The estimated TFAR parameters are then used to calculate an estimate of the innovations signal $e[n]$ from $x[n]$ by inverse filtering, i.e., $\hat{e}[n]=\left(\hat{\mathfrak{H}}_{x}^{-1} x\right)[n]$, where $\hat{\mathbb{H}}_{x}$ is the innovations system corresponding to the estimated intermediate TFAR model.

Compared with the TVMA estimator of [27], this TFMA estimator has a similar accuracy but significantly higher complexity. Thus, we will not consider it any further in the TFMA context. However, we will see in Section VI-D that the method has a good accuracy in the TFARMA context.

\section{Simulation Results}

We compare the TFMA parameter estimator based on the CTFC recursion (see Section IV-B) with the cepstral recursion method for TVMA models proposed in [27]. (The latter method was actually formulated in [27] for the more general TVARMA case.) The TFMA estimator from Section IV-C and the-essentially equivalent-B-TVMA estimator of [7] are not considered in this simulation study because of their significantly higher complexity as discussed above. The CTFC estimates were derived from an EAF estimate of the form (31) that was computed from a single process realization. This EAF estimate was also used for the TVMA technique instead of the evolutionary periodogram [49] used in [27].

We simulated TFMA processes of various lengths $N$ and orders $M_{\mathrm{B}}, L_{\mathrm{B}}$. For each choice of $N, M_{\mathrm{B}}$, and $L_{\mathrm{B}}$, the TFMA parameters were randomly generated such that they were minimum-phase in the sense of [3]. Parameter estimation was then performed for 100 process realizations, using the true orders $M_{\mathrm{B}}, L_{\mathrm{B}}$. From the 100 sets of estimated parameters $\hat{b}_{m, l}^{(i)}, i=1$, $\ldots, 100$, we calculated the normalized mean-square error (MSE)

$$
\mathrm{MSE} \triangleq \frac{1}{100} \sum_{i=1}^{100} \frac{\sum_{m=0}^{M_{\mathrm{B}}} \sum_{l=-L_{\mathrm{B}}}^{L_{\mathrm{B}}}\left|\hat{b}_{m, l}^{(i)}-b_{m, l}\right|^{2}}{\sum_{m=0}^{M_{\mathrm{B}}} \sum_{l=-L_{\mathrm{B}}}^{L_{\mathrm{B}}}\left|b_{m, l}\right|^{2}} .
$$



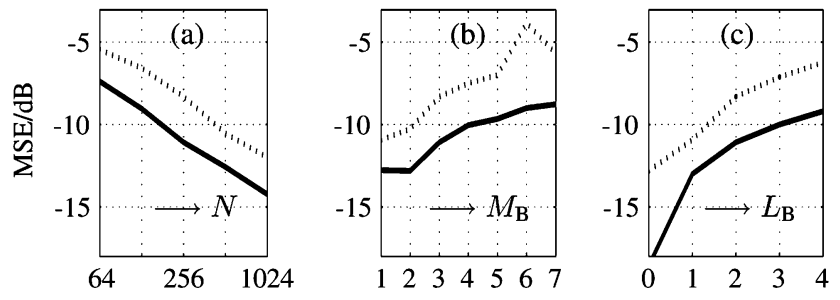

Fig. 3. Normalized MSE of the CTFC-based TFMA estimator (solid line) and the TVMA estimator of [27] (dotted line): (a) $M_{\mathrm{B}}=3, L_{\mathrm{B}}=2, N$ variable; (b) $N=256, L_{\mathrm{B}}=2, M_{\mathrm{B}}$ variable; and (c) $N=256, M_{\mathrm{B}}=3, L_{\mathrm{B}}$ variable.
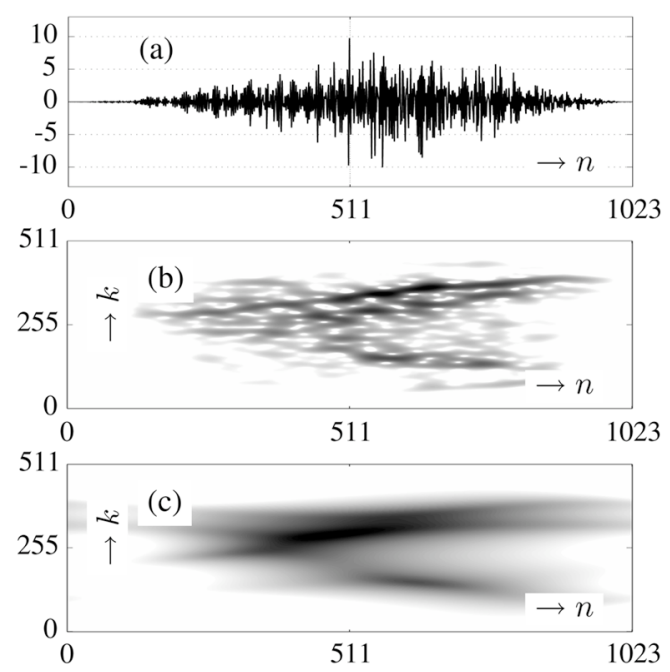

Fig. 4. Spectral analysis of a flat-fading mobile radio channel [50]: (a) Fading coefficient (windowed); (b) smoothed pseudo-Wigner distribution [42], [43]; and (c) TFAR $(8,1 ; 1)$ spectral estimate as defined by $(42)$. Logarithmic grayscale representations are used in (b) and (c).

The results are shown in Fig. 3. The MSE tends to decrease with growing $N$ and increase with $M_{\mathrm{B}}$ and $L_{\mathrm{B}}$; thus, it is lower for TFMA models that are more underspread. Our CTFC-based estimator outperforms the TVMA estimator of [27] by $2-5 \mathrm{~dB}$, which can be explained by the smaller number of parameters estimated.

We furthermore measured the complexities (flop count using Matlab 5.2 implementations) of the two parameter estimators. These complexities account for all computations needed to estimate the model parameters for known model orders $M_{\mathrm{B}}, L_{\mathrm{B}}$ from the basic EAF estimate. Within the simulated ranges of $N, M_{\mathrm{B}}$, and $L_{\mathrm{B}}$, we observed the proposed CTFC method to be about $23 \%$ less complex than the TVMA method.

\section{TFAR MODELING AND PARAMETER ESTIMATION}

In this section, we present the $T F$ autoregressive (TFAR) model and corresponding parameter estimation methods. This model is especially suited for processes whose time-varying spectra exhibit sharp peaks but no deep nulls. An example of a signal that is well modeled by a TFAR model is shown in Fig. 4; this signal consists of several time-varying narrowband components (time-varying spectral peaks).

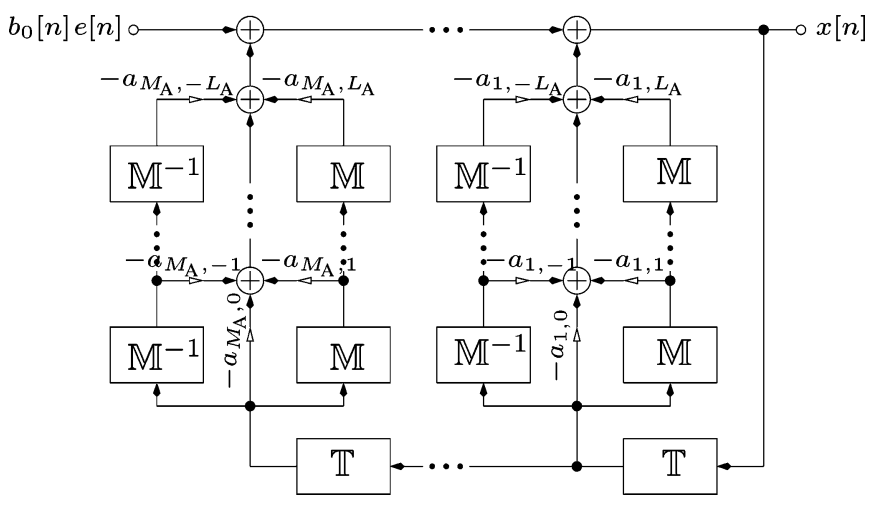

Fig. 5. Block diagram of the $\operatorname{TFAR}\left(M_{\mathrm{A}}, L_{\mathrm{A}} ; L_{\mathrm{B}}\right)$ model. The input is the innovations process $e[n]$ multiplied by the time-dependent amplitude factor $b_{0}[n]=\sum_{l=-L_{\mathrm{B}}}^{L_{\mathrm{B}}} b_{0, l} e^{j \frac{2 \pi}{N} n l}>0$.

\section{A. TFAR Model}

The $\operatorname{TFAR}\left(M_{\mathrm{A}}, L_{\mathrm{A}} ; L_{\mathrm{B}}\right)$ model [1] is defined by the input-output relation

$$
x[n] \triangleq-\sum_{m=1}^{M_{\mathrm{A}}} \sum_{l=-L_{\mathrm{A}}}^{L_{\mathrm{A}}} a_{m, l}\left(\mathbb{S}_{m, l} x\right)[n]+\sum_{l=-L_{\mathrm{B}}}^{L_{\mathrm{B}}} b_{0, l}\left(\mathbb{M}^{l} e\right)[n]
$$

where $e[n]$ is again stationary white noise with variance $1 ; M_{\mathrm{A}}$ and $L_{\mathrm{A}}$ denote the delay and Doppler model orders, respectively; $L_{\mathrm{B}}$ denotes the Doppler model order of a degenerate zero-delay TFMA part; the $N_{\mathrm{A}} \triangleq M_{\mathrm{A}}\left(2 L_{\mathrm{A}}+1\right)$ constants $a_{m, l}$, $m=1, \ldots, M_{\mathrm{A}}, l=-L_{\mathrm{A}}, \ldots, L_{\mathrm{A}}$ are the TFAR parameters; and the $N_{\mathbb{B}_{0}} \triangleq 2 L_{\mathrm{B}}+1$ constants $b_{0, l}, l=-L_{\mathrm{B}}, \ldots, L_{\mathrm{B}}$ are zero-delay TFMA parameters. A block diagram of (36) is depicted in Fig. 5. For $L_{\mathrm{B}}=L_{\mathrm{A}}=0$, the TFAR model reduces to a cyclic form of the time-invariant AR model [5], [6].

The first term in (36), $-\sum_{m=1}^{M_{\mathrm{A}}} \sum_{l=-L_{\mathrm{A}}}^{L_{\mathrm{A}}} a_{m, l}\left(\mathbb{S}_{m, l} x\right)[n]$, is a linear combination of delayed and frequency-shifted versions of $x[n]$. It is a "pure TFAR" component that corresponds to the feedback loop in Fig. 5. The second term, $\sum_{l=-L_{\mathrm{B}}}^{L_{\mathrm{B}}} b_{0, l}\left(\mathbb{M}^{l} e\right)[n]$, is a linear combination of frequency-shifted versions of the innovations process $e[n]$ that corresponds to a degenerate, zero-delay $\operatorname{TFMA}\left(0, L_{\mathrm{B}}\right)$ model. We can write this latter component as $b_{0}[n] e[n]$ with $b_{0}[n]=\sum_{l=-L_{\mathrm{B}}}^{L_{\mathrm{B}}} b_{0, l} e^{j \frac{2 \pi}{N} n l}$. The factor $b_{0}[n]$ models a time-varying input variance $\left|b_{0}[n]\right|^{2}$ that cannot be modeled by the pure TFAR part.

The input-output relation (36) can be expressed as $(A x)[n]=$ $\left(\mathbb{B}_{0} e\right)[n]$ with the causal LTV systems

$$
\mathrm{A} \triangleq \sum_{m=0}^{M_{\mathrm{A}}} \sum_{l=-L_{\mathrm{A}}}^{L_{\mathrm{A}}} a_{m, l} \mathbb{S}_{m, l}, \quad \mathbb{B}_{0} \triangleq \sum_{l=-L_{\mathrm{B}}}^{L_{\mathrm{B}}} b_{0, l} \mathbb{M}^{l}
$$

where $a_{0, l} \triangleq \delta[l]$ (i.e., $\mathrm{A}$ is a monic system). Thus, the innovations system representation of the TFAR process is

$$
x[n]=\left(\mathbb{H}_{x} e\right)[n] \text { with } \mathbb{H}_{x}=\mathbb{A}^{-1} \mathbb{B}_{0} .
$$


We note that the SFs of $A$ and $\mathbb{B}_{0}$ are given by [cf. (23)]

$$
\begin{aligned}
S_{\mathrm{A}}[m, l] & = \begin{cases}N a_{m, l}, & (m, l) \in \mathcal{A} \\
0, & \text { elsewhere }\end{cases} \\
S_{\mathbb{B}_{0}}[m, l] & = \begin{cases}N b_{0, l}, & m=0 \text { and } \\
0, & \text { elsewhere }\end{cases}
\end{aligned}
$$

where $\mathcal{A} \triangleq\left[0, M_{\mathrm{A}}\right] \times\left[-L_{\mathrm{A}}, L_{\mathrm{A}}\right]$.

The TFAR model is a special case of the B-TVARMA model (1), (2) with $M_{\mathrm{B}}=0$, the Fourier (complex exponential) basis $\left\{f_{l}[n]=e^{j 2 \leq \ln / N}\right\}$ [7], and TVAR and (zero-delay) TVMA parameter functions given by

$$
a_{m}[n]=\sum_{l=-L_{\mathrm{A}}}^{L_{\mathrm{A}}} a_{m, l} e^{j \frac{2 \pi}{N} n l}, \quad b_{0}[n]=\sum_{l=-L_{\mathrm{B}}}^{L_{\mathrm{B}}} b_{0, l} e^{j \frac{2 \pi}{N} n l} .
$$

Hence, $a_{m}[n]$ and $b_{0}[n]$ have bandwidth $L_{\mathrm{A}}$ and $L_{\mathrm{B}}$, respectively. Again, our formulation in terms of TF shifts provides a new interpretation and leads to new efficient parameter estimators exploiting the underspread property.

Using the method proposed in [51], the time-varying impulse response $h_{x}[n, m]$ of the TFAR innovations system $\mathbb{H}_{x}$ can be computed with a high complexity of $\mathcal{O}\left(N^{2} M_{\mathrm{A}}^{3}\right)$. However, in the following, we will use an approximate evaluation of $\mathbb{H}_{x}$ that is based on the approximative transfer function calculus described in [35]. In the framework of this calculus, the timevarying transfer function of $\mathbb{H}_{x}=A^{-1} \mathbb{B}_{0}$ is approximated as

$$
Z_{\mathbb{H}_{x}}[n, k] \approx Z_{\mathfrak{H}_{x}}^{(\mathrm{u})}[n, k] \triangleq \frac{Z_{\mathbb{B}_{0}}[n, k]}{Z_{\mathbb{A}}[n, k]}=\frac{b_{0}[n]}{Z_{\text {A }}[n, k]} .
$$

(In practical calculations, this division has to be stabilized, e.g., by replacing the quotient by 0 when $\left|Z_{\mathcal{A}}[n, k]\right|<\delta$ for a suitably chosen threshold $\delta$.) The approximation (41) is justified if the operators $A$ and $\mathbb{B}_{0}$ are jointly underspread, i.e., if $S_{\AA}[m, l]$ and $S_{\mathbb{B}_{0}}[m, l]$ are effectively zero outside a common rectangle about the origin of the $(m, l)$ plane with area much less than $N$ [35], [36]. Because of (38) and (39), this means that $2 M_{\mathrm{A}} L_{\mathrm{A}} \ll N$ and $L_{\mathrm{B}}$ is not much larger than $L_{\mathrm{A}}$. Based on (41), the evolutionary spectrum (9) can be approximated by

$$
\begin{aligned}
G_{x}^{(\mathrm{u})}[n, k] & \triangleq\left|Z_{\mathbb{G}_{x}}^{(\mathrm{u})}[n, k]\right|^{2} \\
& =\left|\frac{\sum_{l=-L_{\mathrm{B}}}^{L_{\mathrm{B}}} b_{0, l} e^{j \frac{2 \pi}{N} n l}}{\sum_{m=0}^{M_{\mathrm{A}}} \sum_{l=-L_{\mathrm{A}}}^{L_{\mathrm{A}}} a_{m, l} e^{j \frac{2 \pi}{N}(n l-k m)}}\right|^{2} .
\end{aligned}
$$

These underspread approximations become exact for $L_{\mathrm{A}}=$ $L_{\mathrm{B}}=0$ (i.e., for a time-invariant $\mathrm{AR}$ model).

\section{B. TFAR Parameter Estimation Based on the TFYW Equations}

We now present a TFAR parameter estimator [1] that generalizes the Yule-Walker method for time-invariant AR models [5], [6] and is a computationally efficient special case of the vector-Yule-Walker method [7]. Calculating the inner product of (36) with $\left(\mathbb{S}_{m, l} x\right)[n]$ and taking expectations yields

$$
\begin{aligned}
& \sum_{m^{\prime}=0}^{M_{\mathrm{A}}} \sum_{l^{\prime}=-L_{\mathrm{A}}}^{L_{\mathrm{A}}} a_{m^{\prime}, l^{\prime}} \mathcal{E}\left\{\left\langle\mathbb{S}_{m^{\prime}, l^{\prime}} x, \mathbb{S}_{m, l} x\right\rangle\right\} \\
&=\sum_{l^{\prime}=-L_{\mathrm{B}}}^{L_{\mathrm{B}}} b_{0, l^{\prime}} \mathcal{E}\left\{\left\langle\mathbb{S}_{0, l^{\prime}} e, \mathbb{S}_{m, l} x\right\rangle\right\}
\end{aligned}
$$

or, equivalently

$$
\begin{aligned}
\sum_{m^{\prime}=0}^{M_{\mathrm{A}}} \sum_{l^{\prime}=-L_{\mathrm{A}}}^{L_{\mathrm{A}}} a_{m^{\prime}, l^{\prime}} A_{x}\left[m-m^{\prime}, l\right. & \left.-l^{\prime}\right] e^{-j \frac{2 \pi}{N} m^{\prime}\left(l-l^{\prime}\right)} \\
& =\sum_{l^{\prime}=-L_{\mathrm{B}}}^{L_{\mathrm{B}}} b_{0, l^{\prime}} A_{e, x}\left[m, l-l^{\prime}\right] .
\end{aligned}
$$

Using the innovations representation (7), it can be shown that $A_{e, x}[m, l]=S_{\mapsto_{x}}^{*}[-m,-l] e^{-j \frac{2 \pi}{N} m l}$. Furthermore, $S_{\mathfrak{H}_{x}}^{*}[-m,-l]=N \delta[m] b_{0,-l}^{*}$ for $m \geq 0$. We thus obtain

$$
\begin{aligned}
\sum_{m^{\prime}=0}^{M_{\mathrm{A}}} \sum_{l^{\prime}=-L_{\mathrm{A}}}^{L_{\mathrm{A}}} a_{m^{\prime}, l^{\prime}} A_{x}\left[m-m^{\prime}, l-l^{\prime}\right] e^{-j \frac{2 \pi}{N} m^{\prime}\left(l-l^{\prime}\right)} \\
=N \delta[m] \sum_{l^{\prime}=-L_{\mathrm{B}}}^{L_{\mathrm{B}}} b_{0, l^{\prime}} b_{0, l^{\prime}-l^{*}}^{*}
\end{aligned}
$$

In what follows, we consider these equations for $m=$ $1, \ldots, M_{\mathrm{A}}$ and $l=-L_{\mathrm{A}}, \ldots, L_{\mathrm{A}}$. Because the right-hand side of (43) vanishes for $m>0$, we obtain (recall that $a_{0, l}=\delta[l]$ )

$$
\begin{array}{r}
\sum_{m^{\prime}=1}^{M_{\mathrm{A}}} \sum_{l^{\prime}=-L_{\mathrm{A}}}^{L_{\mathrm{A}}} a_{m^{\prime}, l^{\prime}} A_{x}\left[m-m^{\prime}, l-l^{\prime}\right] e^{-j \frac{2 \pi}{N} m^{\prime}\left(l-l^{\prime}\right)}=-A_{x}[m, l], \\
m=1, \ldots, M_{\mathrm{A}}, l=-L_{\mathrm{A}}, \ldots, L_{\mathrm{A}} . \quad(44)
\end{array}
$$

These $N_{\text {A }}=M_{\mathrm{A}}\left(2 L_{\mathrm{A}}+1\right)$ linear equations in the $N_{\text {A }}$ unknowns $a_{m, l}$ will be termed the TF Yule-Walker (TFYW) equations. For $L_{\mathrm{A}}=0$, the TFYW equations reduce to the conventional Yule-Walker equations [5], [6].

A compact matrix-vector formulation of the TFYW equations can be obtained by means of a suitable stacking. Let us define the Toeplitz matrices of size $\left(2 L_{\mathrm{A}}+1\right) \times\left(2 L_{\mathrm{A}}+1\right)$, as shown in (45) and (46) at the bottom of the page. Here, the notation

and

$$
\begin{aligned}
\boldsymbol{C}_{m} & =\operatorname{toep}\left\{A_{x}\left[m, 2 L_{\mathrm{A}}\right], A_{x}\left[m, 2 L_{\mathrm{A}}-1\right], \ldots, A_{x}\left[m,-2 L_{\mathrm{A}}\right]\right\} \\
& =\left[\begin{array}{cccc}
A_{x}[m, 0] & A_{x}[m,-1] & \cdots & A_{x}\left[m,-2 L_{\mathrm{A}}\right] \\
A_{x}[m, 1] & A_{x}[m, 0] & \cdots & A_{x}\left[m,-2 L_{\mathrm{A}}+1\right] \\
\vdots & \vdots & \ddots & \vdots \\
A_{x}\left[m, 2 L_{\mathrm{A}}\right] & A_{x}\left[m, 2 L_{\mathrm{A}}-1\right] & \cdots & A_{x}[m, 0]
\end{array}\right], \quad m=-M_{\mathrm{A}}+1, \ldots, M_{\mathrm{A}}-1
\end{aligned}
$$

$$
\boldsymbol{V}_{m}=\operatorname{toep}\left\{e^{j \frac{2 \pi}{N}\left(-2 L_{\mathrm{A}}\right) m}, e^{j \frac{2 \pi}{N}\left(-2 L_{\mathrm{A}}+1\right) m}, \ldots, e^{j \frac{2 \pi}{N} 2 L_{\mathrm{A}} m}\right\}, \quad m=1, \ldots, M_{\mathrm{A}} \text {. }
$$


$\boldsymbol{X}=\operatorname{toep}\left\{x_{1}, x_{2}, \ldots, x_{n}\right\}$ means that the elements of the diagonals of the $n \times n$ Toeplitz matrix $X$ ordered from southwest to northeast are $x_{1}, x_{2}, \ldots, x_{n}$. We also define the $N_{\text {A }} \times N_{\text {A }}$ Toeplitz-block matrix

$$
\boldsymbol{C}=\left[\begin{array}{cccc}
\boldsymbol{C}_{0} \odot \boldsymbol{V}_{1} & \boldsymbol{C}_{-1} \odot \boldsymbol{V}_{2} & \cdots & \boldsymbol{C}_{-M_{\mathrm{A}}+1} \odot \boldsymbol{V}_{M_{\mathrm{A}}} \\
\boldsymbol{C}_{1} \odot \boldsymbol{V}_{1} & \boldsymbol{C}_{0} \odot \boldsymbol{V}_{2} & \cdots & \boldsymbol{C}_{-M_{\mathrm{A}}+2} \odot \boldsymbol{V}_{M_{\mathrm{A}}} \\
\vdots & \vdots & \ddots & \vdots \\
\boldsymbol{C}_{M_{\mathrm{A}}-1} \odot \boldsymbol{V}_{1} & \boldsymbol{C}_{M_{\mathrm{A}}-2} \odot \boldsymbol{V}_{2} & \cdots & \boldsymbol{C}_{0} \odot \boldsymbol{V}_{M_{\mathrm{A}}}
\end{array}\right]
$$

where $\odot$ denotes the Hadamard (elementwise) matrix product. Note that the blocks of $\boldsymbol{C}$ are Toeplitz matrices but their arrangement does not have Toeplitz structure. Finally, we define the following vectors of length $N_{\text {A }}$ :

$\boldsymbol{c}=\left[\boldsymbol{c}_{1}^{T} \cdots \boldsymbol{c}_{M_{\mathrm{A}}}^{T}\right]^{T}$ with $\boldsymbol{c}_{m}=\left[A_{x}\left[m,-L_{\mathrm{A}}\right] \cdots A_{x}\left[m, L_{\mathrm{A}}\right]\right]^{T}$

and

$\boldsymbol{a}=\left[\boldsymbol{a}_{1}^{T} \cdots \boldsymbol{a}_{M_{\mathrm{A}}}^{T}\right]^{T}$ with $\boldsymbol{a}_{m}=\left[a_{m,-L_{\mathrm{A}}} \cdots a_{m, L_{\mathrm{A}}}\right]^{T}$.

The TFYW (44) can then be compactly written as

$$
C a=-c .
$$

This linear equation has to be solved for the TFAR parameter vector $\boldsymbol{a}$.

A different 2-D $\rightarrow$ 1-D stacking leads to alternative TFYW equations with block-Toeplitz structure (i.e., the arrangement of the blocks is Toeplitz but the blocks themselves are not) [1], which allow the use of an efficient solution algorithm with $\mathcal{O}\left(M_{\mathrm{A}}^{3} L_{\mathrm{A}}^{2}\right)$ multiplications [52]. However, the stacking presented above will be shown in Section $\mathrm{V}-\mathrm{C}$ to lead to a parameter estimator that is order-recursive and has a similar complexity.

A TFAR parameter estimator results from the TFYW equations (44) and (50) if the EAF $A_{x}[m, l]$ is replaced by an estimate (see (31) with $\Psi[m, l] \equiv 1$; we note that a TF taper is not required in the TFAR context). This TFAR estimator is similar to the TVAR autocorrelation method [7] for a Fourier basis, except that we use a cyclic estimator of the autocorrelation function $r_{x}[n, m]$ in (31). The difference corresponds to border effects that have little influence on accuracy and complexity.

\section{TFAR Parameter Estimation Based on the Underspread TFYW Equations}

Let us assume that $\mathrm{A}$ is underspread, i.e., $M_{\mathrm{A}} L_{\mathrm{A}} \ll N$. The phase factor $e^{-j \frac{2 \pi}{N} m^{\prime}\left(l-l^{\prime}\right)}$ in (44) can then be approximated by 1 [cf. (11)], whereby the TFYW equations (44) simplify to the underspread TFYW equations

$$
\begin{aligned}
\sum_{m^{\prime}=1}^{M_{\mathrm{A}}} \sum_{l^{\prime}=-L_{\mathrm{A}}}^{L_{\mathrm{A}}} a_{m^{\prime}, l^{\prime}} A_{x}\left[m-m^{\prime}, l-l^{\prime}\right] & =-A_{x}[m, l] \\
m=1, \ldots, M_{\mathrm{A}}, l & =-L_{\mathrm{A}}, \ldots, L_{\mathrm{A}} .
\end{aligned}
$$

In stacked form, these equations read

$$
C^{(\mathrm{u})} a=-c
$$

with the $N_{\text {丹 }} \times N_{\text {丹 }}$ TBT matrix

$$
\begin{aligned}
\boldsymbol{C}^{(\mathrm{u})} & =\operatorname{toep}\left\{\boldsymbol{C}_{M_{\mathrm{A}}-1}, \boldsymbol{C}_{M_{\mathrm{A}}-2}, \ldots, \boldsymbol{C}_{-M_{\mathrm{A}}+1}\right\} \\
& =\left[\begin{array}{cccc}
\boldsymbol{C}_{0} & \boldsymbol{C}_{-1} & \cdots & \boldsymbol{C}_{-M_{\mathrm{A}}+1} \\
\boldsymbol{C}_{1} & \boldsymbol{C}_{0} & \cdots & \boldsymbol{C}_{-M_{\mathrm{A}}+2} \\
\vdots & \vdots & \ddots & \vdots \\
\boldsymbol{C}_{M_{\mathrm{A}}-1} & \boldsymbol{C}_{M_{\mathrm{A}}-2} & \cdots & \boldsymbol{C}_{0}
\end{array}\right]
\end{aligned}
$$

and the vectors $\boldsymbol{c}$ and $\boldsymbol{a}$ as defined in (48) and (49). The underspread TFYW equations reduce to the conventional Yule-Walker equations [5], [6] for $L_{\mathrm{A}}=0$.

The TBT matrix $\boldsymbol{C}^{(\mathrm{u})}$ is an underspread approximation to the Toeplitz-block matrix $\boldsymbol{C}$ in (47) that is obtained by omitting the phase matrices $\boldsymbol{V}_{m}$ in the definition of $\boldsymbol{C}$. Note that $\boldsymbol{C}^{(\mathrm{u})}$ is not a Toeplitz matrix itself, but its blocks $\boldsymbol{C}_{m}$ are Toeplitz matrices and the arrangement of these blocks within $\boldsymbol{C}^{(\mathrm{u})}$ has Toeplitz structure as well. This TBT structure allows the use of the Wax-Kailath algorithm [30] for an efficient solution of the underspread TFYW equations (52) with $\mathcal{O}\left(M_{\mathrm{A}}^{2} L_{\mathrm{A}}^{3}\right)$ multiplications. (The multichannel Levinson algorithm [5] cannot be used because the blocks $\boldsymbol{C}_{m}$ are not symmetric in general.) The recursive structure of the Wax-Kailath algorithm results in an orderrecursive estimator (recursive in the delay order $M_{\mathrm{A}}$ ). Thus, we are able to recursively estimate all $\operatorname{TFAR}\left(M_{\mathrm{A}}, L_{\mathrm{A}} ; L_{\mathrm{B}}\right)$ models with $M_{\mathrm{A}}$ ranging from 1 to a prescribed maximum TFAR order. The savings in complexity due to the underspread approximation will be assessed experimentally in Section V-E.

Again, a different 2-D $\rightarrow$ 1-D stacking leads to an alternative TBT form of the underspread TFYW equations for which the Wax-Kailath algorithm requires $\mathcal{O}\left(M_{\mathrm{A}}^{3} L_{\mathrm{A}}^{2}\right)$ multiplications. Even though this is the same complexity order as for the exact TFYW equations with alternative stacking [1], the actual complexity is reduced by a factor of 2 . However, the stacking we used is advantageous in that the Wax-Kailath algorithm here results in an order-recursive estimator, which is not true for the alternative stacking [1].

\section{Estimation of the Zero-Delay TFMA Parameters}

It remains to estimate the parameters $b_{0, l}$ of the degenerate $\operatorname{TFMA}\left(0, L_{\mathrm{B}}\right)$ component. This can be done by means of the CTFC relation (30). An alternative method is related to the TFYW approach and does not require calculation of the CTFC. Recall that the parameters $b_{0, l}$ model the positive time-varying innovations amplitude $b_{0}[n]=\sum_{l=-L_{\mathrm{B}}}^{L_{\mathrm{B}}} b_{0, l} e^{j \frac{2 \pi}{N} n l}$ [cf. (40)]. The time-varying innovations variance $b_{0}^{2}[n]$ can be expressed as

$$
b_{0}^{2}[n]=\sum_{l=-2 L_{\mathrm{B}}}^{2 L_{\mathrm{B}}} \sigma_{l} e^{j \frac{2 \pi}{N} n l}
$$

with

$$
\sigma_{l} \triangleq \sum_{l^{\prime}=-L_{\mathrm{B}}}^{L_{\mathrm{B}}} b_{0, l^{\prime}} b_{0, l^{\prime}-l}^{*} .
$$



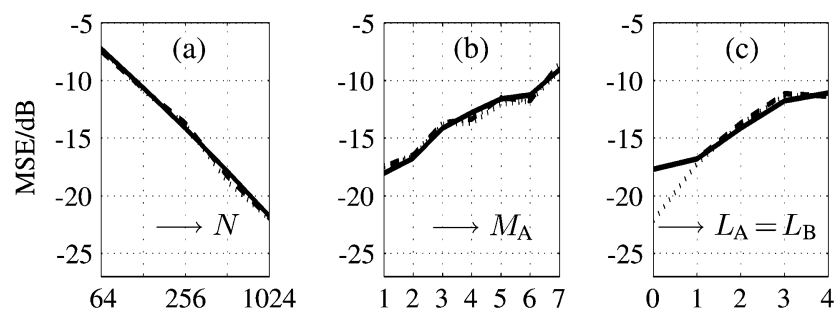

Fig. 6. Normalized MSE of the TFAR estimator based on the underspread TFYW equations (solid line), the TFAR estimator based on the exact TFYW equations (dashed line), and the B-TVAR covariance method [7] (dotted line): (a) $M_{\mathrm{A}}=3, L_{\mathrm{A}}=L_{\mathrm{B}}=2, N$ variable; (b) $N=256, L_{\mathrm{A}}=L_{\mathrm{B}}=2, M_{\mathrm{A}}$ variable; and (c) $N=256, M_{\mathrm{A}}=3, L_{\mathrm{A}}=L_{\mathrm{B}}$ variable.

The equations (43) for $m=0$ and $l=-2 L_{\mathrm{B}}, \ldots, 2 L_{\mathrm{B}}$ can be written in terms of the $\sigma_{l}$ 's as

$$
\begin{array}{r}
\sigma_{l}=\frac{1}{N} \sum_{m^{\prime}=0}^{M_{\mathrm{A}}} \sum_{l^{\prime}=-L_{\mathrm{A}}}^{L_{\mathrm{A}}} a_{m^{\prime}, l^{\prime}} A_{x}\left[-m^{\prime}, l-l^{\prime}\right] e^{-j \frac{2 \pi}{N} m^{\prime}\left(l-l^{\prime}\right)}, \\
l=-2 L_{\mathrm{B}}, \ldots, 2 L_{\mathrm{B}} .
\end{array}
$$

This shows how the $\sigma_{l}$ 's can be calculated from the (previously estimated) TFAR parameters $a_{m, l}$. We can then calculate $b_{0}^{2}[n]$ via (54) and, thus, obtain $b_{0}[n] \geq 0$. Finally, the $b_{0, l}$ 's are computed by inversion of (40). Alternatively, one can use spectral factorization techniques [53] to directly compute the $b_{0, l}$ 's from the $\sigma_{l}$ 's.

\section{E. Simulation Results}

We compare the TFYW method (Sections V-B and V-D), the underspread TFYW method (Sections V-C and V-D), and the B-TVAR covariance method of [7] using a Fourier basis. We simulated TFAR processes with various $N, M_{\mathrm{A}}$, and $L_{\mathrm{A}}=L_{\mathrm{B}}$. The TFAR parameters were generated randomly such that they were stable in the sense of [3]. They were then estimated from a single process realization $x[n]$ using the true model orders. This estimation was carried out for 100 realizations, and the normalized MSE [cf. (35)] was calculated. Fig. 6 shows that the MSEs of all estimators are quite similar, apart from a smaller MSE of the B-TVAR method for small Doppler order. As in the TFMA case, the MSE is lower for models that are more underspread. Fig. 7 shows the computational savings (measured in \% of flops) of the underspread TFYW method relative to the exact TFYW method and the B-TVAR covariance method of [7] (we note that the complexities of these latter two methods are effectively equal). These savings are seen to depend on $N$, $M_{\mathrm{A}}$, and $L_{\mathrm{A}}=L_{\mathrm{B}}$; they are about $30 \%$ on average but can be as high as $40 \%$. They are due to the fact that the inversion of a TBT matrix required by the underspread TFYW method is about twice as fast as the inversion of a block-Toeplitz matrix required by the B-TVAR method.

\section{TFARMA MODELING AND PARAMETER ESTIMATION}

The TFARMA model is a combination of the TFMA and TFAR models. It is able to model both time-varying spectral nulls and time-varying spectral peaks. An example of a signal suited to TFARMA modeling will be considered in Section VII.
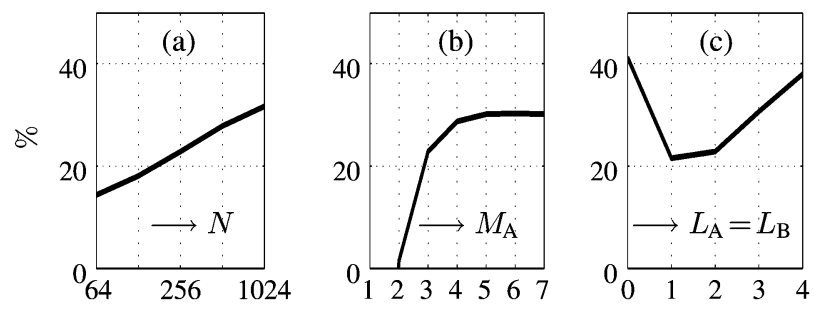

Fig. 7. Flop savings of the TFAR estimator based on the underspread TFYW equations relative to the TFAR estimator based on the exact TFYW equations (the complexity of this latter estimator is effectively equal to that of the B-TVAR covariance method of [7]): (a) $M_{\mathrm{A}}=3, L_{\mathrm{A}}=L_{\mathrm{B}}=2, N$ variable; (b) $N=256, L_{\mathrm{A}}=L_{\mathrm{B}}=2, M_{\mathrm{A}}$ variable; and (c) $N=256, M_{\mathrm{A}}=3$, $L_{\mathrm{A}}=L_{\mathrm{B}}$ variable.

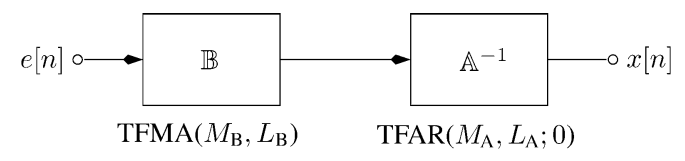

Fig. 8. Block diagram of the TFARMA $\left(M_{\mathrm{A}}, L_{\mathrm{A}} ; M_{\mathrm{B}}, L_{\mathrm{B}}\right)$ model.

\section{A. TFARMA Model}

A TFARMA $\left(M_{\mathrm{A}}, L_{\mathrm{A}} ; M_{\mathrm{B}}, L_{\mathrm{B}}\right)$ process is defined as [3]

$x[n] \triangleq-\sum_{m=1}^{M_{\mathrm{A}}} \sum_{l=-L_{\mathrm{A}}}^{L_{\mathrm{A}}} a_{m, l}\left(\mathbb{S}_{m, l} x\right)[n]+\sum_{m=0}^{M_{\mathrm{B}}} \sum_{l=-L_{\mathrm{B}}}^{L_{\mathrm{B}}} b_{m, l}\left(\mathbb{S}_{m, l} e\right)[n]$

where $e[n]$ is again stationary white noise with variance 1. As shown in Fig. 8, the $\operatorname{TFARMA}\left(M_{\mathrm{A}}, L_{\mathrm{A}} ; M_{\mathrm{B}}, L_{\mathrm{B}}\right)$ model is a concatenation of a $\operatorname{TFMA}\left(M_{\mathrm{B}}, L_{\mathrm{B}}\right)$ model and a $\operatorname{TFAR}\left(M_{\mathrm{A}}, L_{\mathrm{A}} ; 0\right)$ model. It is characterized by the $N_{\mathbb{B}}=\left(M_{\mathrm{B}}+1\right)\left(2 L_{\mathrm{B}}+1\right)$ TFMA parameters $b_{m, l}, m=$ $0, \ldots, M_{\mathrm{B}}, l=-L_{\mathrm{B}}, \ldots, L_{\mathrm{B}}$ and the $N_{\mathrm{A}}=M_{\mathrm{A}}\left(2 L_{\mathrm{A}}+1\right)$ TFAR parameters $a_{m, l}, m=1, \ldots, M_{\mathrm{A}}, l=-L_{\mathrm{A}}, \ldots, L_{\mathrm{A}}$. For $L_{\mathrm{A}}=L_{\mathrm{B}}=0$, the TFARMA model reduces to a cyclic version of the time-invariant $\operatorname{ARMA}\left(M_{\mathrm{A}}, M_{\mathrm{B}}\right)$ model [5], [6]. Further special cases are the TFMA $\left(M_{\mathrm{B}}, L_{\mathrm{B}}\right)$ model (obtained for $\left.M_{\mathrm{A}}=L_{\mathrm{A}}=0\right)$ and the $\operatorname{TFAR}\left(M_{\mathrm{A}}, L_{\mathrm{A}} ; L_{\mathrm{B}}\right)$ model (obtained for $M_{\mathrm{B}}=0$ ).

The TFARMA model is a special case of the B-TVARMA model (1), (2) with the Fourier (complex exponential) basis $\left\{f_{l}[n]=e^{j 2 \pi \ln / N}\right\}$ [7]. The TVAR parameters $a_{m}[n]$ and TVMA parameters $b_{m}[n]$ are given by (40) and (24), respectively; they are band-limited with bandwidth $L_{\mathrm{A}}$ and $L_{\mathrm{B}}$, respectively.

The input-output relation (55) can be written as $(A x)[n]=$ $(\mathbb{B e})[n]$ with the causal LTV systems $\mathbb{A}$ and $\mathbb{B}$ given by (37) and (22), respectively. The innovations system representation of $x[n]$ is thus obtained as

$$
x[n]=\left(\mathbb{H}_{x} e\right)[n] \quad \text { with } \quad \mathbb{H}_{x}=\mathrm{A}^{-1} \mathbb{B} .
$$

The nonzero values of the SF of $\mathbb{A}$ and $\mathbb{B}$ are given by $N a_{m, l}$ and $N b_{m, l}$, respectively [cf. (38) and (23)].

If the operators $\mathbb{A}$ and $\mathbb{B}$ are jointly underspread (see Section V-A), the time-varying transfer function of the 
TFARMA innovations system $\mathbb{H}_{x}=\mathbb{A}^{-1} \mathbb{B}$ can be approximated as

$$
Z_{\mathbb{H}_{x}}[n, k] \approx Z_{\mathbb{H}_{x}}^{(\mathrm{u})}[n, k] \triangleq \frac{Z_{\mathbb{B}}[n, k]}{Z_{\mathbb{A}}[n, k]}
$$

which generalizes (41). An underspread approximation of the evolutionary spectrum $G_{x}[n, k]$ is then given by

$$
\begin{aligned}
G_{x}^{(\mathrm{u})}[n, k] & \triangleq\left|Z_{\mathbb{H}_{x}}^{(\mathrm{u})}[n, k]\right|^{2} \\
& =\left|\frac{\sum_{m=0}^{M_{\mathrm{B}}} \sum_{l=-L_{\mathrm{B}}}^{L_{\mathrm{B}}} b_{m, l} e^{j \frac{2 \pi}{N}(n l-k m)}}{\sum_{m=0}^{M_{\mathrm{A}}} \sum_{l=-L_{\mathrm{A}}}^{L_{\mathrm{A}}} a_{m, l} e^{j \frac{2 \pi}{N}(n l-k m)}}\right|^{2}
\end{aligned}
$$

where $a_{0, l} \triangleq \delta[l]$.

\section{B. Estimation of the TFAR Part}

The TFAR parameters $a_{m, l}$ can be estimated by extensions of the TFYW methods discussed in Sections V-B and V-C. Computing the inner product of $(55)$ with $\left(\mathbb{S}_{m, l} x\right)[n]$ and taking expectations yields [cf. (43) and (33)]

$$
\begin{aligned}
& \sum_{m^{\prime}=0}^{M_{\mathrm{A}}} \sum_{l^{\prime}=-L_{\mathrm{A}}}^{L_{\mathrm{A}}} a_{m^{\prime}, l^{\prime}} A_{x}\left[m-m^{\prime}, l-l^{\prime}\right] e^{-j \frac{2 \pi}{N} m^{\prime}\left(l-l^{\prime}\right)} \\
& =\sum_{m^{\prime}=0}^{M_{\mathrm{B}}} \sum_{l^{\prime}=-L_{\mathrm{B}}}^{L_{\mathrm{B}}} b_{m^{\prime}, l^{\prime}} A_{e, x}\left[m-m^{\prime}, l-l^{\prime}\right] e^{-j \frac{2 \pi}{N} m^{\prime}\left(l-l^{\prime}\right)}
\end{aligned}
$$

where $A_{e, x}[m, l]=\mathcal{E}\left\{\left\langle e, \mathbb{S}_{m, l} x\right\rangle\right\}=S_{\mathbb{H}_{x}}^{*}[-m,-l] e^{-j \frac{2 \pi}{N} m l}$. Because $S_{\uplus_{x}}[m, l]=0$ for $m<0$, the right-hand side of (58) vanishes for $m>M_{\mathrm{B}}$. Restricting to $m=M_{\mathrm{B}}+1, \ldots, M_{\mathrm{B}}+$ $M_{\mathrm{A}}$ and $l=-L_{\mathrm{A}}, \ldots, L_{\mathrm{A}}$, we thus obtain the following set of equations that do not contain the TFMA parameters $b_{m, l}$ [cf. (44)]:

$$
\begin{gathered}
\sum_{m^{\prime}=1}^{M_{\mathrm{A}}} \sum_{l^{\prime}=-L_{\mathrm{A}}}^{L_{\mathrm{A}}} a_{m^{\prime}, l^{\prime}} A_{x}\left[m-m^{\prime}, l-l^{\prime}\right] e^{-j \frac{2 \pi}{N} m^{\prime}\left(l-l^{\prime}\right)}=-A_{x}[m, l], \\
m=M_{\mathrm{B}}+1, \ldots, M_{\mathrm{B}}+M_{\mathrm{A}}, l=-L_{\mathrm{A}}, \ldots, L_{\mathrm{A}} .
\end{gathered}
$$

These $N_{\text {A }}=M_{\mathrm{A}}\left(2 L_{\mathrm{A}}+1\right)$ linear equations in the $N_{\text {A }}$ unknowns $a_{m, l}$ will be termed the extended TFYW equations. They can be written as

$$
\boldsymbol{C}_{\mathrm{e}} \boldsymbol{a}=-\boldsymbol{c}_{\mathrm{e}}
$$

with the $N_{\text {A }} \times N_{\text {A }}$ Toeplitz-block matrix [cf. (47)] shown at the bottom of the page, where $\boldsymbol{C}_{m}$ and $\boldsymbol{V}_{m}$ were defined in (45) and (46), respectively. Furthermore, $\boldsymbol{c}_{\mathrm{e}}$ is a length- $N_{\mathrm{A}}$ vector defined as $c_{\mathrm{e}}=\left[\boldsymbol{c}_{M_{\mathrm{B}}+1}^{T} \cdots \boldsymbol{c}_{M_{\mathrm{B}}+M_{\mathrm{A}}}^{T}\right]^{T}$ with $\boldsymbol{c}_{m}=\left[A_{x}\left[m,-L_{\mathrm{A}}\right] \cdots A_{x}\left[m, L_{\mathrm{A}}\right]\right]^{T}$, and $\boldsymbol{a}$ is as in (49). The resulting estimator is essentially equivalent, up to border effects, to the time-varying extended YW method of [7]. For $L_{\mathrm{A}}=0$, the extended TFYW equations reduce to the conventional extended Yule-Walker equations [6].

If $\mathrm{A}$ is underspread, i.e., $M_{\mathrm{A}} L_{\mathrm{A}} \ll N$, we can again approximate the phase factor $e^{-j \frac{2 \pi}{N} m^{\prime}\left(l-l^{\prime}\right)}$ in (59) by 1 . This yields the underspread extended TFYW equations [cf. (51)]

$$
\begin{aligned}
& \sum_{m^{\prime}=1}^{M_{\mathrm{A}}} \sum_{l^{\prime}=-L_{\mathrm{A}}}^{L_{\mathrm{A}}} a_{m^{\prime}, l^{\prime}} A_{x}\left[m-m^{\prime}, l-l^{\prime}\right]=-A_{x}[m, l], \\
& m=M_{\mathrm{B}}+1, \ldots, M_{\mathrm{B}}+M_{\mathrm{A}}, l=-L_{\mathrm{A}}, \ldots, L_{\mathrm{A}}
\end{aligned}
$$

or compactly $C_{\mathrm{e}}^{(\mathrm{u})} \boldsymbol{a}=-\boldsymbol{c}_{\mathrm{e}}$, with the $N_{\AA} \times N_{\AA}$ TBT matrix $\boldsymbol{C}_{\mathrm{e}}^{(\mathrm{u})}=\operatorname{toep}\left\{\boldsymbol{C}_{M_{\mathrm{B}}+M_{\mathrm{A}}-1}, \boldsymbol{C}_{M_{\mathrm{B}}+M_{\mathrm{A}}-2}, \ldots, \boldsymbol{C}_{M_{\mathrm{B}}-M_{\mathrm{A}}+1}\right\}$ [cf. (53)]. These TBT equations can again be solved with $\mathcal{O}\left(M_{\mathrm{A}}^{2} L_{\mathrm{A}}^{3}\right)$ multiplications by means of the Wax-Kailath algorithm. This results in an order-recursive estimator (recursive in the delay order $M_{\mathrm{A}}$ ).

\section{Estimation of the TFMA Part}

Next, we present a CTFC-based estimator for the TFMA parameters $b_{m, l}$. As in Section IV-B, we extend the time-invariant approach of [40] to derive a cepstral recursion for the TFMA parameters $b_{m, l}$ that involves the cyclic CTFC $\mathfrak{S}_{\mathbb{B}}[m, l]$. We assume that $\mathbb{A}$ and $\mathbb{B}$ are jointly underspread. Recall that the time-varying transfer function $Z_{\mathbb{H}_{x}}[n, k]$ equals $Z_{\mathbb{H}_{x}}(u, z)$ in (12) evaluated on the unit bicircle, i.e., for $u=e^{j 2 \pi n / N}$ and $z=e^{j 2 \pi k / N}$. We can extend the underspread approximation (56) to a small neighborhood $\mathcal{N}$ of the unit bicircle, as follows:

$$
\begin{aligned}
Z_{\mathbb{H}_{x}}(u, z) & \approx Z_{\mathbb{H}_{x}}^{(\mathrm{u})}(u, z) \triangleq \frac{Z_{\mathbb{B}}(u, z)}{Z_{\mathbb{A}}(u, z)} \\
& =\frac{\sum_{m=0}^{M_{\mathrm{B}}} \sum_{l=-L_{\mathrm{B}}}^{L_{\mathrm{B}}} b_{m, l} u^{l} z^{-m}}{\sum_{m=0}^{M_{\mathrm{A}}} \sum_{l=-L_{\mathrm{A}}}^{L_{\mathrm{A}}} a_{m, l} u^{l} z^{-m}}, \quad(u, z) \in \mathcal{N} .
\end{aligned}
$$

This extension to $\mathcal{N}$ is justified because the numerator $Z_{\mathbb{B}}(u, z)$ and denominator $Z_{\AA}(u, z)$ are polynomial functions and thus do not vary rapidly when we move a little away from the unit bicircle. A corresponding underspread approximation of the CTFC, denoted $\widetilde{\mathfrak{S}}_{\uplus_{x}}^{(\mathrm{u})}[m, l]$, is then defined according to (13), i.e.,

$$
\log \left(Z_{\uplus_{x}}^{(\mathrm{u})}(u, z)\right)=\frac{1}{N} \sum_{m=0}^{\infty} \sum_{l=-\infty}^{\infty} \tilde{\mathfrak{S}}_{\mathbb{H}_{x}}^{(\mathrm{u})}[m, l] u^{l} z^{-m} .
$$

We insert (60) and proceed similarly as in Section IV-B by differentiating the result with respect to $z^{-1}$ and approximating $\tilde{\mathfrak{S}}_{\mathbb{H}_{x}}^{(\mathrm{u})}[m, l]$ by the cyclic underspread CTFC

$$
\boldsymbol{C}_{\mathrm{e}}=\left[\begin{array}{cccc}
\boldsymbol{C}_{M_{\mathrm{B}}} \odot \boldsymbol{V}_{1} & \boldsymbol{C}_{M_{\mathrm{B}}-1} \odot \boldsymbol{V}_{2} & \cdots & \boldsymbol{C}_{M_{\mathrm{B}}-M_{\mathrm{A}}+1} \odot \boldsymbol{V}_{M_{\mathrm{A}}} \\
\boldsymbol{C}_{M_{\mathrm{B}}+1} \odot \boldsymbol{V}_{1} & \boldsymbol{C}_{M_{\mathrm{B}}} \odot \boldsymbol{V}_{2} & \cdots & \boldsymbol{C}_{M_{\mathrm{B}}-M_{\mathrm{A}}+2} \odot \boldsymbol{V}_{M_{\mathrm{A}}} \\
\vdots & \vdots & \ddots & \vdots \\
\boldsymbol{C}_{M_{\mathrm{B}}+M_{\mathrm{A}}-1} \odot \boldsymbol{V}_{1} & \boldsymbol{C}_{M_{\mathrm{B}}+M_{\mathrm{A}}-2} \odot \boldsymbol{V}_{2} & \cdots & \boldsymbol{C}_{M_{\mathrm{B}}} \odot \boldsymbol{V}_{M_{\mathrm{A}}}
\end{array}\right]
$$


$\mathfrak{S}_{\sharp_{x}}^{(\mathrm{u})}[m, l]=\sum_{i=-\infty}^{\infty} \sum_{i^{\prime}=-\infty}^{\infty} \tilde{\mathfrak{S}}_{\mathbb{H}_{x}}^{(\mathrm{u})}\left[m+i N, l+i^{\prime} N\right] . \mathrm{We}$ then use $\mathfrak{S}_{\mathfrak{H}_{x}}^{(\mathrm{u})}[m, l] \approx \mathfrak{S}_{\mathbb{H}_{x}}[m, l]$ and (20) to obtain the following CTFC recursion for $b_{m, l}$ :

$$
\begin{aligned}
& b_{m, l}= \sum_{m^{\prime}=1}^{m-1} \sum_{l^{\prime}=-L}^{L} \frac{m-m^{\prime}}{m}\left(a_{m-m^{\prime}, l-l^{\prime}} b_{m^{\prime}, l^{\prime}}\right. \\
&\left.-a_{m^{\prime}, l^{\prime}} b_{m-m^{\prime}, l-l^{\prime}}\right) \\
&+\frac{1}{N} \sum_{m^{\prime}=0}^{m-1} \sum_{l^{\prime}=-L_{\mathrm{A}}}^{L_{\mathrm{A}}} \frac{m-m^{\prime}}{m} \mathfrak{A}_{x}\left[m-m^{\prime}, l-l^{\prime}\right] \\
& \times \sum_{m^{\prime \prime}=0}^{m^{\prime}} \sum_{l^{\prime \prime}=-L_{\mathrm{A}}}^{L_{\mathrm{A}}} a_{m^{\prime \prime}, l^{\prime \prime}} b_{m^{\prime}-m^{\prime \prime}, l^{\prime}-l^{\prime \prime}} \\
&+\sum_{l^{\prime}=-L_{\mathrm{A}}}^{L_{\mathrm{A}}} a_{m, l^{\prime}} b_{0, l-l^{\prime}}, \\
& \quad m=1, \ldots, M_{\mathrm{B}}, l=-L_{\mathrm{B}}, \ldots, L_{\mathrm{B}}
\end{aligned}
$$

with $L \triangleq \max \left\{L_{\mathrm{B}}, L_{\mathrm{A}}\right\}$. This recursion can be initialized by (30). It allows us to calculate the TFMA parameters $b_{m, l}$, assuming that the TFAR parameters $a_{m, l}$ were previously calculated as discussed in Section VI-B.

Finally, $\mathfrak{A}_{x}[m, l]$ has to be replaced by an estimate that is derived from an EAF estimate of the form (31). The resulting ( $M_{\mathrm{B}}$-recursive) estimator of the TFMA part is less complex than the cepstral estimator of [27], which estimates $N\left(M_{\mathrm{A}}+\right.$ $\left.M_{\mathrm{B}}+1\right)$ parameters via the evolutionary periodogram [47] and the time-varying innovations variance by a separate procedure (cf. our discussion in Section IV-B). For $L_{\mathrm{A}}=L_{\mathrm{B}}=0$, our estimator reduces to the method for time-invariant ARMA models presented in [40], but with a more sophisticated initialization.

\section{Simulation Results}

We simulated two different TFARMA parameter estimators. For estimation of the TFAR part, both estimators use the underspread extended TFYW method of Section VI-B with an additional stabilization as discussed in [3]. For estimation of the TFMA part, one estimator uses the extended CTFC-based method of Section VI-C, while the other uses the TFMA method based on an intermediate high-order TFAR model discussed in Section IV-C. As a reference method, we also simulated the cepstral recursion method for TVARMA models proposed in [27], which estimates all $N\left(M_{\mathrm{A}}+M_{\mathrm{B}}+1\right)$ TVARMA parameters $a_{m}[n]$ and $b_{m}[n]$. (The B-TVARMA method of [7] is not considered in this simulation study. This is because, as mentioned in Section VI-B, it is equivalent up to border effects to our TFARMA method using the TFYW technique for estimation of the TFAR part and an intermediate high-order TFAR model for estimation of the TFMA part.)

These three estimation methods were applied to TFARMA processes with various lengths $N$ and orders $M_{\mathrm{A}}=M_{\mathrm{B}}+1$ and $L_{\mathrm{A}}=L_{\mathrm{B}}$. For each process, the TFARMA parameters were estimated from a single process realization $x[n]$, using the true model orders. This was repeated for 100 realizations, and the normalized MSE was calculated. From Fig. 9, it is seen that our methods outperform the method of [27] by up to $5 \mathrm{~dB}$, because of the smaller number of parameters to be estimated.
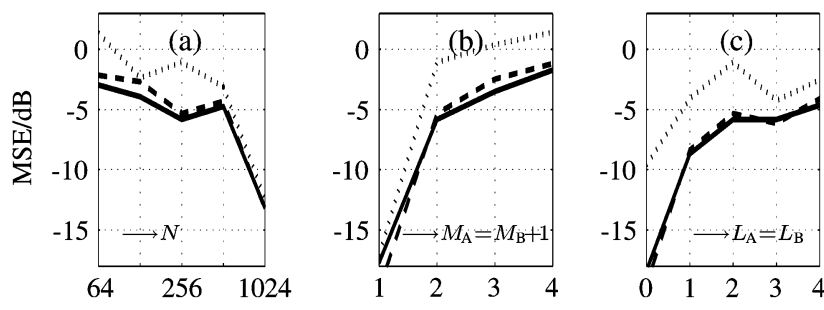

Fig. 9. Normalized MSE of TFARMA and TVARMA parameter estimators: (a) $M_{\mathrm{A}}=2, M_{\mathrm{B}}=1, L_{\mathrm{A}}=L_{\mathrm{B}}=2, N$ variable; (b) $N=256, L_{\mathrm{A}}=$ $L_{\mathrm{B}}=2, M_{\mathrm{A}}=M_{\mathrm{B}}+1$ variable; and (c) $N=256, M_{\mathrm{A}}=2, M_{\mathrm{B}}=$ $1, L_{\mathrm{A}}=L_{\mathrm{B}}$ variable. Solid line: extended underspread TFYW-based TFAR estimator and extended CTFC-based TFMA estimator, dashed line: extended underspread TFYW-based TFAR estimator and TFMA estimator based on an intermediate high-order TFAR model; dotted line: TVARMA estimator of [27].

Furthermore, we observed that, within the simulated ranges of $N, M_{\mathrm{A}}=M_{\mathrm{B}}+1$, and $L_{\mathrm{A}}=L_{\mathrm{B}}$, the estimator using the extended CTFC-based method is about $36 \%$ less complex than the TVARMA method of [27] (cf. Section IV-D). The estimator using an intermediate high-order TFAR model has a similar MSE performance as the estimator using the extended CTFCbased method. We also note that it is similar, with respect to both MSE performance and complexity, to the B-TVARMA method of [7] (not shown in Fig. 9); however, both the estimator using an intermediate high-order TFAR model and the B-TVARMA method of [7] are significantly more complex than our CTFC-based method.

\section{APPLICATION EXAMPLE}

In previous sections, we studied the accuracy of the proposed TFAR, TFMA, and TFARMA parameter estimators by applying them to signals synthetically generated according to the respective model. We will now apply the TFAR, TFMA, and TFARMA models and, for each model, the best parameter estimator to the time-varying spectral analysis of the quasi-natural signal shown in Fig. 10(a). This signal, of length $N=512$, is the sum of two echolocation chirp signals emitted by a Daubenton's bat (http:// www.londonbats.org.uk). A smoothed pseudo-Wigner distribution (SPWD) [42], [43] of this signal is shown in Fig. 10(b).

We performed TFAR, TFMA, and TFARMA analyses on this signal using the parameter estimators indicated in Table I. From the estimated TFAR, TFMA, or TFARMA parameters, we computed the corresponding parametric spectral estimates, i.e., estimates of the evolutionary spectrum $G_{x}[n, k]$ [TFMA case, see (25)] or of its underspread approximation $G_{x}^{(\mathrm{u})}[n, k]$ [TFAR and TFARMA cases, see (42) and (57), respectively]. The model orders were estimated by means of the AIC [3], [54]; the resulting orders are indicated in Table I. All parameters were stabilized by means of the technique described in [3], with stabilization parameter $\lambda=0.95$.

The spectral estimates are depicted in Fig. 10(c)-(e). It is seen that the TFAR spectrum displays the two chirp components fairly well, although there are some spurious peaks (this effect is well known from AR models [5]) and the overall resolution is poorer than that of the nonparametric SPWD in Fig. 10(b). The TFMA spectrum, as expected, is unable to resolve the timevarying spectral peaks of the signal. Finally, the TFARMA spectrum exhibits better resolution than the SPWD, and it does not 
TABLE I

Model Orders Estimated and Parameter Estimation Methods USED for the Two-Component Bat SignaL

\begin{tabular}{|l||l|l|}
\hline Model & Estimated model orders & Parameter estimation method(s) used \\
\hline \hline TFAR & $M_{\mathrm{A}}=9, L_{\mathrm{A}}=1, L_{\mathrm{B}}=1$ & $\begin{array}{l}\text { TFAR parameters: underspread TFYW estimator (Section V-C); } \\
\text { zero-delay } \text { TFMA parameters: TFYW-related estimator (Section V-D) }\end{array}$ \\
\hline TFMA & $M_{\mathrm{B}}=5, L_{\mathrm{B}}=3$ & CTFC-based estimator (Section IV-B) \\
\hline TFARMA & $M_{\mathrm{A}}=5, L_{\mathrm{A}}=1, M_{\mathrm{B}}=4, L_{\mathrm{B}}=1$ & $\begin{array}{l}\text { TFAR parameters: underspread TFYW estimator (Section VI-B); } \\
\text { TFMA parameters: CTFC-based estimator (Section VI-C) }\end{array}$ \\
\hline
\end{tabular}
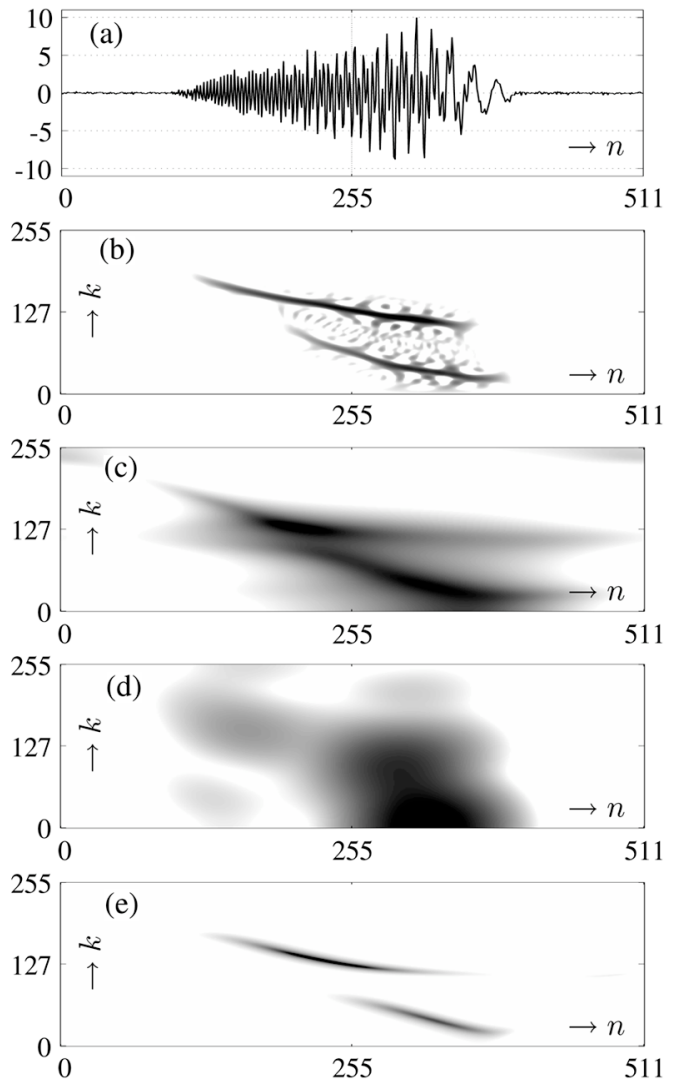

Fig. 10. Time-varying parametric spectral analysis of the sum of two bat echolocation signals: (a) Time-domain signal; (b) smoothed pseudo-Wigner distribution; (c) TFAR $(9,1 ; 1)$ spectral estimate; (d) TFMA $(5,3)$ spectral estimate; and (e) TFARMA $(5,1 ; 4,1)$ spectral estimate. The parameter estimators employed are indicated in Table I. Logarithmic gray-scale representations are used in (b)-(e).

contain any cross terms as does the SPWD [43]; on the other hand, the TF localization of the components deviates slightly from that in the SPWD. It should be noted at this point that these parametric spectra involve only 30 (TFAR and TFARMA) or 42 (TFMA) parameters.

\section{CONCLUSION}

TFARMA models for nonstationary random processes, with TFAR and TFMA models as special cases, were presented and shown to be a TF-symmetric reformulation of time-varying ARMA (AR, MA) models using a Fourier basis. This reformulation is physically intuitive because it uses time shifts (delays) and frequency shifts to model the nonstationary dynamics of a process. TFARMA (TFAR, TFMA) models are parsimonious for the practically relevant class of processes with a limited time-frequency correlation structure (underspread processes).
For estimating the parameters of TFARMA, TFAR, and TFMA models, we proposed methods that are based on a novel time-frequency cepstrum or on time-frequency Yule-Walker equations. In either case, the parameter estimators are order-recursive with respect to the delay order. Some of our estimators rely on "underspread approximations" that exploit the underspread property to achieve a reduction of computational complexity. Our simulation results demonstrated that the proposed methods outperform existing methods for time-varying ARMA (AR, MA) modeling in terms of accuracy and/or complexity. The application of the proposed methods to the time-varying spectral analysis of a quasi-natural signal showed that the TFARMA spectral estimate is able to improve on the smoothed pseudo-Wigner distribution in terms of resolution and absence of cross terms, even though it involves only a small number of parameters.

\section{ACKNOWLEDGMENT}

The authors would like to thank W. Mecklenbräuker and C. Mecklenbräuker for helpful suggestions and stimulating discussions. They are also grateful to the anonymous reviewers for numerous comments that have led to an improvement of this paper.

\section{REFERENCES}

[1] M. Jachan, G. Matz, and F. Hlawatsch, "Time-frequency-autoregressive random processes: Modeling and fast parameter estimation," in Proc. IEEE Int. Conf. Acoustics, Speech, Signal Processing (ICASSP), Hong Kong, Apr. 2003, vol. VI, pp. 125-128.

[2] M. Jachan, G. Matz, and F. Hlawatsch, "Time-frequency-moving-average random processes: Principles and cepstral methods for parameter estimation," in Proc. IEEE Int. Conf. Acoustics, Speech, Signal Processing (ICASSP), Montreal, QC, Canada, May 2004, vol. II, pp. 757-760.

[3] M. Jachan, G. Matz, and F. Hlawatsch, "TFARMA models: Order estimation and stabilization," in Proc. IEEE Int. Conf. Acoustics, Speech, Signal Processing (ICASSP), Philadelphia, PA, Mar. 2005, vol. IV, pp. 301-304.

[4] M. Jachan, F. Hlawatsch, and G. Matz, "Linear methods for TFARMA parameter estimation and system approximation," in Proc. 13th IEEE Workshop Statistical Signal Processing, Bordeaux, France, Jul. 2005, pp. 909-914.

[5] S. M. Kay, Modern Spectral Estimation. Englewood Cliffs, NJ: Prentice-Hall, 1988.

[6] P. Stoica and R. Moses, Introduction to Spectral Analysis. Englewood Cliffs, NJ: Prentice-Hall, 1997.

[7] Y. Grenier, "Time-dependent ARMA modeling of nonstationary signals," IEEE Trans. Acoust., Speech, Signal Process., vol. 31, pp. 899-911, Aug. 1983.

[8] T. S. Rao, "The fitting of non-stationary time-series models with timedependent parameters," J. Roy. Stat. Soc. Ser. B, vol. 32, no. 2, pp. 312-322, 1970.

[9] L. A. Liporace, "Linear estimation of nonstationary signals," J. Acoust. Soc. Amer., vol. 58, pp. 1288-1295, Dec. 1975. 
[10] M. Juntunen and J. P. Kaipio, "Stabilization of smoothness priors deterministic regression TVAR models," in Proc. IEEE Int. Conf. Acoustics, Speech, Signal Processing (ICASSP), Istanbul, Turkey, Jun. 2000, pp. $568-571$.

[11] M. Niedźwiecki, Identification of Time-Varying Processes. New York: Wiley, 2000.

[12] K. M. Malladi, R. V. R. Kumar, and K. V. Rao, "A Gauss-Markov model formulation for the estimation of ARMA model of time-varying signals and systems," in Proc. IEEE-SP Int. Sympos. Time-Frequency Time-Scale Analysis, Pittsburgh, PA, 1998, pp. 657-660.

[13] Y. Grenier, "Time varying lattices and autoregressive models: Parameter estimation," in Proc. IEEE Int. Conf. Acoustics, Speech, Signal Processing (ICASSP), Paris, France, May 1982, pp. 1337-1340.

[14] Y. Grenier, "Estimation of non-stationary moving-average models," in Proc. IEEE IEEE Int. Conf. Acoustics, Speech, Signal Processing (ICASSP), Boston, MA, 1983, pp. 268-271.

[15] M. G. Hall, A. V. Oppenheim, and A. S. Willsky, "Time-varying parametric modeling of speech," Signal Process., vol. 5, pp. 267-285, May 1983.

[16] Y. Grenier, "Non-stationary signal modelling with application to bat echolocation calls," ACUSTICA, vol. 61, pp. 156-165, Sep. 1986.

[17] Y. Grenier, "Parametric time-frequency representations," in Traitement du Signal/Signal Processing, Les Houches, Session XLV, J. L. Lacoume, T. S. Durrani, and R. Stora, Eds. Amsterdam, The Netherlands: Elsevier, 1987, pp. 338-397.

[18] M. E. Yüksel, S. Kara, and N. Taşpinar, "Time-dependent ARMA modeling of continuous wave ultrasonic Doppler signals," in Proc. IEEE-SP Workshop Statistical Signal Array Processing, Corfu, Greece, Jun. 1996, pp. 260-263.

[19] T. Robert and C. Mailhes, "Continuously evolving classification using time-varying AR modeling," in Proc. IEEE-SP Int. Symp. Time-Frequency Time-Scale Analysis, Paris, France, 1996, pp. 241-244.

[20] M. K. Tsatsanis and G. B. Giannakis, "Subspace methods for blind estimation of time-varying FIR channels," IEEE Trans. Signal Process., vol. 45, no. 12, pp. 3084-3093, Dec. 1997.

[21] Y. Grenier, "Nonstationary ARMA models via simultaneous AR and MA estimation," in Proc. IEEE Int. Conf. Acoustics, Speech, Signal Processing (ICASSP), Tokyo, Japan, Apr. 1986, vol. 11, pp. 2339-2342.

[22] E. Moulines, P. Duhamel, J.-F. Cardoso, and S. Mayrargue, "Subspace methods for the blind identification of multichannel FIR filters," IEEE Trans. Signal Process., vol. 43, no. 2, pp. 516-525, Feb. 1995.

[23] G. B. Giannakis and C. Tepedelenlioğlu, "Basis expansion models and diversity techniques for blind identification and equalization of time-varying channels," Proc. IEEE, vol. 86, pp. 1969-1986, Oct. 1998.

[24] A. M. Sayeed and B. Aazhang, "Joint multipath-Doppler diversity in mobile wireless communications," IEEE Trans. Commun., vol. 47, no. 1, pp. 123-132, Jan. 1999.

[25] I. Barhumi, G. Leus, and M. Moonen, "Time-varying FIR decision feedback equalization of doubly-selective channels," in Proc. IEEE GLOBECOM, San Francisco, CA, Dec. 2003, pp. 2263-2268.

[26] I. Barhumi, G. Leus, and M. Moonen, "Time-varying FIR equalization of doubly-selective channels," in Proc. IEEE Int. Conf. Communications (ICC), Ottawa, ON, Canada, May 2003, pp. 3246-3250.

[27] A. Kaderli and A. S. Kayhan, "Spectral estimation of nonstationary ARMA processes using the evolutionary cepstrum," IEEE Signal Process. Lett., vol. 9, pp. 130-132, Apr. 2002.

[28] G. Matz and F. Hlawatsch, "Nonstationary spectral analysis based on time-frequency operator symbols and underspread approximations," IEEE Trans. Inf. Theory, vol. 52, pp. 1067-1086, Mar. 2006.

[29] G. Matz and F. Hlawatsch, "Time-varying power spectra of nonstationary random processes," in Time-Frequency Signal Analysis and Processing: A Comprehensive Reference, B. Boashash, Ed. Oxford, U.K.: Elsevier, 2003, ch. 9.4, pp. 400-409.

[30] M. Wax and T. Kailath, "Efficient inversion of Toeplitz-block Toeplitz matrix," IEEE Trans. Acoust., Speech, Signal Process., vol. 31, pp. 1218-1221, Oct. 1983

[31] N. A. Abdrabbo and M. B. Priestley, "On the prediction of nonstationary processes," J. Roy. Stat. Soc. Ser. B, vol. 29, no. 3, pp. 570-585, 1967.

[32] P. A. Bello, "Characterization of randomly time-variant linear channels," IEEE Trans. Commun. Syst., vol. 11, pp. 360-393, 1963.

[33] G. Matz and F. Hlawatsch, "Time-frequency transfer function calculus of linear time-varying systems," in Time-Frequency Signal Analysis and Processing: A Comprehensive Reference, B. Boashash, Ed. Oxford, U.K.: Elsevier, 2003, ch. 4.7, pp. 135-144.
[34] L. A. Zadeh, "Frequency analysis of variable networks," Proc. IRE, vol. 76, pp. 291-299, Mar. 1950.

[35] G. Matz and F. Hlawatsch, "Time-frequency transfer function calculus (symbolic calculus) of linear time-varying systems (linear operators) based on a generalized underspread theory," J. Math. Phys. (Special Issue on Wavelet and Time-Frequency Analysis), vol. 39, pp. 4041-4071, Aug. 1998.

[36] W. Kozek, "On the transfer function calculus for underspread LTV channels," IEEE Trans. Signal Process., vol. 45, no. 1, pp. 219-223, Jan. 1997.

[37] H. Cramér, "On some classes of nonstationary stochastic processes," in Proc. 4th Berkeley Symp. Math. Stat. Prob., 1961, pp. 57-78, Univ. Calif. Press.

[38] M. B. Priestley, "Evolutionary spectra and non-stationary processes," J. Roy. Stat. Soc. Ser. B, vol. 27, no. 2, pp. 204-237, 1965.

[39] D. Tjøstheim, "Spectral generating operators for non-stationary processes," Adv. Appl. Prob., vol. 8, pp. 831-846, 1976.

[40] A. Kaderli and A. S. Kayhan, "Spectral estimation of ARMA processes using ARMA-cepstrum recursion," IEEE Signal Process. Lett., vol. 7, pp. 259-261, Sep. 2000.

[41] A. V. Oppenheim and R. W. Schafer, Digital Signal Processing. Englewood Cliffs, NJ: Prentice-Hall, 1975.

[42] P. Flandrin, Time-Frequency/Time-Scale Analysis. San Diego, CA: Academic, 1999

[43] F. Hlawatsch and P. Flandrin, "The interference structure of the Wigner distribution and related time-frequency signal representations," in The Wigner Distribution-Theory and Applications in Signal Processing, W. Mecklenbräuker and F. Hlawatsch, Eds. Amsterdam, The Netherlands: Elsevier, 1997, pp. 59-133.

[44] W. Kozek and K. Riedel, "Quadratic time-varying spectral estimation for underspread processes," in Proc. IEEE-SP Int. Symp. Time-Frequency Time-Scale Analysis, Philadelphia, PA, Oct. 1994, pp. 460-463.

[45] W. Kozek, H. G. Feichtinger, and J. Scharinger, "Matched multiwindow methods for the estimation and filtering of nonstationary processes," in Proc. IEEE Int. Symp. Circuits Systems (ISCAS), Atlanta, GA, May 1996, pp. 509-512.

[46] J. Durbin, "Efficient estimation of parameters in moving-average models," Biometrika, vol. 46, pp. 306-316, 1959.

[47] P. M. T. Broersen, "The best order of long autoregressive models for moving average estimation," in Proc. EUSIPCO, Trieste, Italy, Sep. 1996, pp. 799-802.

[48] P. M. T. Broersen, "Autoregressive model orders for Durbin's MA and ARMA estimators," IEEE Trans. Signal Process., vol. 48, no. 8, pp. 2454-2457, Aug. 2000.

[49] A. S. Kayhan, A. El-Jaroudi, and L. F. Chaparro, "Evolutionary periodogram for nonstationary signals," IEEE Trans. Signal Process., vol. 42, no. 6, pp. 1527-1536, Jun. 1994.

[50] G. Matz, "Characterization of non-WSSUS fading dispersive channels," in Proc. IEEE Int. Conf. Communications (ICC), Anchorage, AK, May 2003, pp. 2480-2484.

[51] N.-C. Huang and J. K. Aggarwal, "On linear shift-variant digital filters," IEEE Trans. Circuits Syst., vol. 27, pp. 672-679, Aug. 1980.

[52] H. Akaike, "Block Toeplitz matrix inversion," SIAM J. Appl. Math., vol. 24, pp. 234-241, Mar. 1973.

[53] A. H. Sayed and T. Kailath, "A survey of spectral factorization methods," Numer. Linear Algebra Appl., vol. 8, pp. 467-496, 2001.

[54] H. Akaike, "A new look at the statistical model identification," IEEE Trans. Autom. Control, vol. 19, pp. 716-723, Dec. 1974.

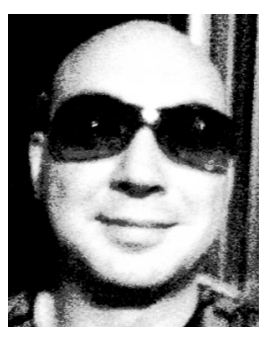

Michael Jachan received the M.Sc. and Ph.D. degrees in telecommunications/signal processing from Vienna University of Technology, Vienna, Austria, in 2001 and 2006, respectively.

From December 2001 to May 2002, he was with the ftw. Telecommunications Research Center Vienna, where he was working on an xDSL system simulator. From June 2002 to July 2006, he was with the Institute of Communications and Radio-Frequency Engineering, Vienna University of Technology. Since November 2006, he has been with the Freiburg Center for Data Analysis and Modeling (FDM), Freiburg University, Germany, where he is working on signal processing methods for medical applications. His research interests are in applied statistical signal processing. 


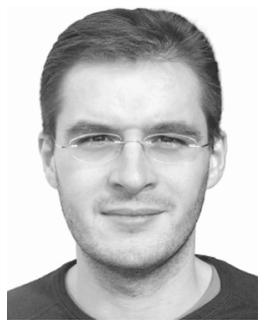

Gerald Matz (S'95-M'01-SM'07) received the Dipl.-Ing. and Dr. Techn. degrees, both in electrical engineering, and the Habilitation degree for communication systems, all from the Vienna University of Technology, Austria, in 1994, 2000, and 2004, respectively.

Since 1995, he has been with the Institute of Communications and Radio-Frequency Engineering, Vienna University of Technology, where he currently holds a tenured position as Associate Professor. From March 2004 to February 2005, he was on leave as an Erwin Schrödinger Fellow with the Laboratoire des Signaux et Systèmes, Ecole Supérieure d'Electricité, France. He has published approximately 90 papers in international journals, conference proceedings, and edited books .His research interests include wireless communications, statistical signal processing, and information theory.

Prof. Matz has directed or actively participated in several research projects funded by the Austrian Science Fund (FWF) and by the European Union. He serves as Associate Editor of the IEEE TRANSACTIONS ON SigNAL PROCESSING and the IEEE Signal Processing LeTters, was Technical Program Co-Chair of the 12th European Signal Processing Conference, and member of the Program Committee of numerous IEEE conferences. In 2006, he received the Kardinal Innitzer Most Promising Young Investigator Award.

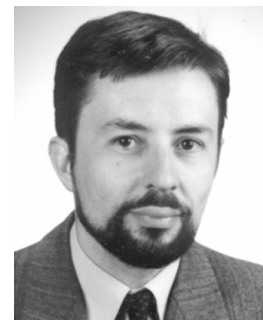

Franz Hlawatsch (S'85-M'88-SM'00) received the Diplom-Ingenieur, Dr. Techn., and Univ.-Dozent (habilitation) degrees in electrical engineering/signal processing from the Vienna University of Technology, Vienna, Austria in 1983, 1988, and 1996, respectively.

Since 1983, he has been with the Institute of Communications and Radio-Frequency Engineering, Vienna University of Technology, where he holds an Associate Professor position. During 1991-1992, as a recipient of an Erwin Schrödinger Fellowship, he spent a sabbatical year with the Department of Electrical Engineering, University of Rhode Island, Kingston, RI. In 1999, 2000, and 2001, he held one-month Visiting Professor positions with INP/ENSEEIHT/TeSA, Toulouse, France, and IRCCyN, Nantes, France. He (co)authored a book, a review paper that appeared in the IEEE Signal Processing Magazine, about 150 refereed scientific papers and book chapters, and two patents. He coedited two books. His research interests include signal processing for wireless communications, nonstationary statistical signal processing, and time-frequency signal processing.

Prof. Hlawatsch was Technical Program Co-Chair of EUSIPCO 2004 and served on the technical committees of numerous IEEE conferences. From 2003 to 2007, he served as Associate Editor for the IEEE TRANSACTIONS ON SIGNAL PROCESSING. Since 2004, he has been a member of the IEEE SPCOM Technical Committee. He is coauthor of a paper that won an IEEE Signal Processing Society Young Author Best Paper Award. 